\title{
Phase space methods for Majorana fermions
}

\author{
Ria Rushin Joseph ${ }^{1}$, Laura E C Rosales-Zárate ${ }^{1,2}$ and \\ Peter D Drummond ${ }^{1}$ \\ ${ }^{1}$ Centre for Quantum and Optical Science, Swinburne University of Technology, \\ Melbourne 3122, Australia \\ ${ }^{2}$ Centro de Investigaciones en Óptica A.C., León, Guanajuato 37150, México \\ E-mail: pdrummond@swin.edu.au
}

\begin{abstract}
Fermionic phase space representations are a promising method for studying correlated fermion systems. The fermionic Q-function and P-function have been defined using Gaussian operators of fermion annihilation and creation operators. The resulting phase-space of covariance matrices belongs to the symmetry class D, one of the non-standard symmetry classes. This was originally proposed to study mesoscopic normal-metal-superconducting hybrid structures, which is the type of structure that has led to recent experimental observations of Majorana fermions. Under a unitary transformation, it is possible to express these Gaussian operators using real anti-symmetric matrices and Majorana operators, which are much simpler mathematical objects. We derive differential identities involving Majorana fermion operators and an antisymmetric matrix which are relevant to the derivation of the corresponding Fokker-Planck equations on symmetric space. These enable stochastic simulations either in real or imaginary time. This formalism has direct relevance to the study of fermionic systems in which there are Majorana type excitations, and is an alternative to using expansions involving conventional Fermi operators. The approach is illustrated by showing how a linear coupled Hamiltonian as used to study topological excitations can be transformed to Fokker-Planck and stochastic equation form, including dissipation through particle losses.

PACS numbers: 03.75.Ss, 67.85.Lm, 24.10.Cn, 03.65.Ca, 03.65.Fd
\end{abstract}

Submitted to: J. Phys. A: Math. Gen. 


\section{Introduction}

Due to the complexity arising from Pauli's exclusion principle, Fermi statistics are a challenging topic in theoretical physics. As a result, new approaches to understanding fermion physics are important. While ideas from coherence theory play an important role in the physics of bosons, they have not yet been widely used to study fermions. Probabilistic methods are especially interesting, as they have the promise that they can be efficiently sampled. Here we show, by using fermionic Q-functions, that first order Fokker Plank equations can be obtained both for linear coupling and for dissipation. This makes simulations possible in regimes where topological excitations and Majorana fermions are expected to occur.

The control acquired in experiments of ultra-cold fermionic atomic systems opens new areas that were not explored previously, including Majorana fermions [1] 3] and the unitary limit of strong interactions [4. These complement studies of strongly correlated fermions in condensed matter physics, which are now investigating such exotic types of fermionic excitation as well.

In particular, there has been a recent resurgence of interest in the work of Majorana [1. As well as the question of fundamental Majorana fermions, which is still not completely resolved, there is a growing interest in Majorana-like excitations and quasi-particles in condensed matter physics [5]. There have been several claims of experimental observations, typically in environments involving coupling of semiconductors to superconductors [6 6 ]. Recent reviews regarding Majorana fermions focus on their possible observations in nuclear, particle and solid states physics [3, 10, or their possible relation to Random Matrix Theory [11.

Some of this work is also closely related to proposals for topological insulators and quantum computing [12]. While this development is still speculative [13, 14, it does reveal an important issue. How can one evaluate topological or related quantum technology proposals? Many studies of this type omit crucial issues such as decoherence mechanisms. To understand decoherence and fidelity, which are essential to quantum technology applications, a method for treating such Hamiltonian terms theoretically is needed. Neither mean field theory nor perturbation theory is sufficient to treat these deeply quantum-mechanical systems. The use of exact methods on an orthogonal basis lacks scalability; thus, other methods will be needed.

The general field of Majorana fermionic excitations in condensed matter systems is growing [2]. The common problem that occurs theoretically is that if one wishes to go beyond mean field theory, then either one must work in a restricted Hilbert space of small dimension, or else there are sign issues from using Monte-Carlo methods. Recent methods in this direction, intended to overcome the sign problem, include using the symmetry of the Hamiltonian properties of Lie groups and Lie algebras [15] or treating fermionic systems interacting with spins [16]. A different sign free approach is to consider the Majorana representation together with Quantum Monte Carlo simulations [17-19]. Finally we point out that extremely powerful methods for classical simulation of fermion 
chains with linear couplings are known to exist [20 22], originally based on work by Valiant [23], although this technique has not been used to treat particle loss or gain.

Just as the use of a coherent state basis and the corresponding phase-space methods proved essential to understanding laser physics [24], it is useful to develop a similar coherent formalism for Majorana physics. Phase space representations have been widely used in quantum optics [25, 26] and ultracold atoms [27, 28] in order to study dynamics. The advantage of using phase-space methods is that it is possible to obtain an exact Fokker Planck equation from the time evolution equation of the density operator. The Fokker Planck equation can be readily transformed to a stochastic differential equation [29,30]. An advantage of this method is that it is possible to sample the stochastic equation probabilistically, allowing one to perform quantum dynamical calculations for large number of modes and particles [31.

These methods can be used to treat dissipation and losses. Such processes occur in many physical systems and represent, for example, the loss of atoms from a trap in the case of magnetically trapped atoms. For electrons they may originate in the transfer of electrons from one band to another. In general, such physical processes are part of the quantum dynamics, and are described by the use of master equations. Since these represent non-unitary processes on the subsystem of interest, they require appropriate techniques able to represent the entire density matrix. This is shown here through a simple example.

The purpose of these approaches is to enable the use of probabilistic methods, which can overcome the exponential growth problem of large many-body Hilbert spaces. An example is the positive Q-function. For this representation, there have been applications using the $\mathrm{SU}(2)$ coherent states [32, 33], including simulations of Bell violations [34]. Another application of the dynamics of the Q-function was in the study of quantum correlations of a degenerate optical parametric oscillator [35].

These methods have been recently extended to the fermionic raising and lowering operators, resulting in fermionic analogs of the normally ordered P-function and the anti-normally ordered Q-function for bosons. Such phase space representations use as a basis the Gaussian operators [36]38], which are exponential of quadratic forms of creation and annihilation operators. A related concept is the fermionic Gaussian state, for which the density matrix is an exponential of quadratic forms of operators defined by a correlation matrix. Some applications of the fermionic Gaussian operators include the study of the Fermi Hubbard model [38,40, and fermionic molecular dissociation [41,42].

It was proved recently that the fermionic Q-function exists as a complete probabilistic representation [43]. Hence, it is important to develop this method further. Earlier theoretical work used a Gaussian basis of Fermi operators [36 38]. By transforming the basis in terms of Majorana operators, the resulting differential identities can help to treat Majorana physics also.

The fermionic Gaussian operators that we consider belong to the non-standard symmetry class D defined by Altland and Zirnbauer [44]. This non-standard symmetry class was originally proposed to study mesoscopic normal-metal-superconducting hybrid 
structures, which is exactly the type of structure that has led to recent experimental observations of Majorana quasi-particles. We show that, under a unitary transformation, we can write the class D operators in terms of an anti-symmetric matrix and Majorana variables [1, and obtain a positive phase-space representation. Since the distribution is now defined in terms of the Majorana operators, we expect it will be useful in investigating the recently discovered Majorana fermionic quasi-particles in hybrid superconductor or ultra-cold atomic systems [6, 7, 45]. Other symmetries can also be used to identify classes of operator transformations, and to reduce the resulting dimensionality. These are not treated here, but allow this work to be extended.

There has been much work exploring the connection of Class D symmetry with topological and Majorana-like excitations [10,46 53]. This relationship therefore has a strong physical underpinning. Moreover, the use of conventional methods such as mean field theory is not applicable when there are strong quantum correlations. Therefore, our approach is to investigate other mathematical relations involving differential identities, that can help these investigations in future. These identities allow the symmetry class to be utilized in the operator representation. The end result is that one can treat exponentially complex Hilbert spaces as dynamical equations in a continuous phasespace, which are generally simpler to solve.

In this communication, we derive differential identities for the Gaussian phase space representation using Majorana operators. These identities are relevant to the general use of a Gaussian basis and to the fermionic Q and P-function. Differential identities allow simulation of the time evolution either in real or imaginary time. The latter approach has already been used to investigate the ground state of the Fermi Hubbard model [39,54]. Thus, these differential identities are potentially relevant to the dynamics of Majorana systems or to systems that have Majorana operator excitations. We expect that using methods based on Majorana operators will further help to study other Fermi systems as well, because the class D symmetry has a fundamentally simpler form using these operators.

This paper is organized as follows: In section 2 we review the Gaussian phase space representation as well as we introduced the Majorana Gaussian operators. Section 3 presents our main result, which is the differential identities for the Majorana operators. These are given for the un-normalized and normalized form of the Majorana Gaussian operator. We obtain identities for both ordered and unordered Majorana operator products. In section 4 we present the corresponding phase space representations with Fermi and Majorana operators. We present the time evolution equation for the Majorana Q-function for one of these differential equations in section 5. In section 6, we discuss the time evolution of the Q-function for an open quantum system. Finally, a summary of our results and conclusions are given in section 7 . 


\section{Fermionic Gaussian operators}

Fermionic phase space representations that use a phase-space of Grassmann variables are known [55]. While this is useful for analytic calculations, it is an exponentially complex problem to directly represent the Grassmann variables computationally. Therefore, in order to avoid such exponential complexity, it is helpful to map the fermion problem into a phase-space of covariances, which are more computationally tractable. One method to achieve this is to use the fermionic Gaussian operators as a basis [36, 37, 43, 56]. In this case, the phase space is a space of covariance matrices, which are complex variables. We emphasise that through using identities, any correlation order can be calculated, including the extremely high orders that can occur in some quantum technology and mesoscopic physics applications [34,57]. An alternative method is to derive the covariance matrix equations of motion from Grassmann variable equations [58], which is not treated here.

In this section, we review the properties of the fermionic Gaussian operators in the notation used in the paper. These were first introduced as variational BCS states [59, 60], and their algebraic properties as operator transformations have been studied extensively [44,61. We consider a fermionic system that can be decomposed into $M$ modes, where $\hat{\boldsymbol{a}}$ is defined as a vector of $M$ annihilation operators, while $\hat{\boldsymbol{a}}^{\dagger}$ is the corresponding vector of $M$ creation operators. The Fermi operators, $\hat{a}_{i}$ and $\hat{a}_{j}^{\dagger}$, obey the usual anti commutation relations:

$$
\left\{\hat{a}_{i}, \hat{a}_{j}^{\dagger}\right\}=\delta_{i j}, \quad\left\{\hat{a}_{i}, \hat{a}_{j}\right\}=0 .
$$

The single mode number operator is $\hat{n}=\widehat{a}^{\dagger} \widehat{a}$, and the action of the operators on the single-mode number states $|n\rangle$ is that $\widehat{a}^{\dagger}|n\rangle=(1-n)|n+1\rangle$, and $\widehat{a}|n\rangle=n|n-1\rangle$.

Using the Fermi operators, we define a $2 M$ extended vector of creation and annihilation operators $\underline{\hat{a}}=\left(\hat{\boldsymbol{a}}^{T}, \hat{\boldsymbol{a}}^{\dagger}\right)^{T}$, with the corresponding adjoint vector defined as $\underline{\hat{a}}^{\dagger}=\left(\hat{\boldsymbol{a}}^{\dagger}, \hat{\boldsymbol{a}}^{T}\right)=\left(\hat{a}_{1}^{\dagger}, \ldots, \hat{a}_{M}^{\dagger}, \hat{a}_{1}, \ldots, \hat{a}_{M}\right)$. We use the following notation throughout the paper: $M$ vectors are in bold type as $\hat{\boldsymbol{a}}, 2 M$ vectors are denoted with a single underline as $\underline{\hat{a}}, M \times M$ matrices are in bold type as $\mathbf{I}$, and $2 M \times 2 M$ matrices are denoted with a double underline as $\underline{\underline{I}}$.

The un-normalized fermionic Gaussian operators $\hat{\Lambda}_{f}^{u}$ are defined here [36, 37] as the set of all possible normally-ordered Gaussian functions of these $2 M$ dimensional operator vectors,

$$
\hat{\Lambda}_{f}^{u}(\underline{\underline{\mu}})=: \exp \left[-\underline{\hat{a}}^{\dagger} \underline{\underline{\mu}} \underline{\hat{a}} / 2\right]: .
$$

Similar definitions were subsequently introduced elsewhere [62, 63], except without normal ordering. Throughout the paper, normal ordering applies to the annihilation and creation operators, and is denoted by : ... . This includes a sign change for every fermion operator swap, hence $: \hat{a}_{i} \hat{a}_{j}^{\dagger}:=-\hat{a}_{j}^{\dagger} \hat{a}_{i}$. Anti-normal ordering is denoted by $\{\ldots\}$, hence $\left\{\hat{a}_{j}^{\dagger} \hat{a}_{i}\right\}=-\hat{a}_{i} \hat{a}_{j}^{\dagger}$. We will also use nested orderings [36]. In this case the outer 
ordering does not reorder the inner one, but the sign changes still take place for each swap - for example $\left\{: \hat{O} \hat{b}_{i}^{\dagger}: \hat{b}_{j}\right\}=-\hat{b}_{j} \hat{b}_{i}^{\dagger} \hat{O}$. A convenient notation is to introduce a basis of unit trace fermionic operators $\hat{\Lambda}_{f}$, where:

$$
\hat{\Lambda}_{f}=\sqrt{\operatorname{det}[\underline{\mathrm{i} \sigma} \underline{\underline{ }}]}: \exp \left[-\underline{\hat{a}}^{\dagger}\left(\underline{\underline{\sigma}}^{-1}-2 \underline{\underline{J}}\right) \underline{\hat{a}} / 2\right]: \text {. }
$$

Here $\underline{\underline{J}}$ is a matrix square root of the identity:

$$
\underline{=}=\left[\begin{array}{cc}
-\mathbf{I} & 0 \\
0 & \mathbf{I}
\end{array}\right]
$$

The importance of expressing the Gaussian operators in this form is that these Gaussian operators using annihilation and creation operators are the basis for the fermionic $\mathrm{Q}$ and P-functions. The matrices $\mathbf{0}$ and $\mathbf{I}$ are the $M \times M$ zero and identity matrices, respectively, and $\underline{\underline{\sigma}}=(\underline{\underline{\mu}}+2 \underline{\underline{J}})^{-1}$ is the covariance matrix, which can be expressed in terms of an $M \times M$ matrix $\mathbf{n}$ and a complex antisymmetric $M \times M$ matrix m:

$$
\underline{\underline{\sigma}}=(\underline{\underline{\mu}}+2 \underline{\underline{J}})^{-1}=\left[\begin{array}{cc}
\mathbf{n}^{T}-\mathbf{I} & \mathbf{m} \\
\mathbf{m}^{+} & \mathbf{I}-\mathbf{n}
\end{array}\right] .
$$

We use the notation $\mathbf{n}^{T}$ to indicate a transpose, $\mathbf{m}^{*}$ a complex conjugate, and $\mathbf{m}^{\dagger}$ a conjugate transpose. The most general fermionic Gaussian operator is non-hermitian. In this case $\mathbf{n}$ is not hermitian, and $\mathbf{m}^{+}, \mathbf{m}$ are independent antisymmetric complex matrices. To obtain hermitian Gaussian operators, one must impose the additional restrictions that $\mathbf{m}^{+}=\mathbf{m}^{\dagger}$ and $\mathbf{n}=\mathbf{n}^{\dagger}$ [37]. The $\boldsymbol{n}$ and $\boldsymbol{m}$ notation is used because these terms in the covariance matrix of a hermitian Gaussian density matrix are equal to the normal and anomalous Green's functions, which are ubiquitous in fermionic many-body theory. We note that the covariance matrix correspond to the first-order correlations of the Gaussian operators in normally ordered form, since $\operatorname{Tr}\left[: \underline{\hat{a}}^{\dagger} \hat{\Lambda}_{f}\right]=\underline{\underline{\sigma}}[36]$.

The Gaussian operators have a more widespread utility than just their obvious value in treating Gaussian states, which are the ground state solutions of quadratic Hamiltonians. The general fermionic Gaussian operators are a complete basis for all fermionic density matrices, including those that are not Gaussian, through taking linear combinations of Gaussian operators. On the other hand, the hermitian fermionic Gaussian operators can be regarded as physical density matrices by themselves.

Several nonstandard symmetry classes were defined by Altland and Zirnbauer [44]. Here we focus on Class D, which corresponds to cases which do not have timereversal or spin-rotation symmetry. The general hermitian Gaussian operators have the nonstandard Class D symmetry [43].

\subsection{Majorana Gaussian operators}

The work of Majorana [1] showed that a more symmetric form of quantization was obtainable if the Fermi operators are transformed to an hermitian Majorana fermion 
operator basis. We use the normalization that is most common in modern papers, where the Majorana operators are obtained using a matrix transformation of the usual Fermi annihilation and creation operators [3],

$$
\hat{\hat{\gamma}}=\left[\begin{array}{c}
\hat{\gamma}_{(1)} \\
\hat{\gamma}_{(2)}
\end{array}\right]=\underline{\underline{U_{0}}} \underline{\underline{\hat{a}}} .
$$

This uses a matrix $\underline{\underline{U_{0}}}$, given by [61]:

$$
\underline{\underline{U_{0}}}=\left[\begin{array}{cc}
\mathbf{I} & \mathbf{I} \\
-\mathrm{i} \mathbf{I} & \mathrm{i} \mathbf{I}
\end{array}\right] \text {, }
$$

which is not unitary, since $\underline{\underline{U_{0}}}{ }^{\dagger}=2{\underline{\underline{U_{0}}}}^{-1}$, although $\underline{\underline{U_{0}}} / \sqrt{2}$ is unitary. This definition corresponds to introducing hermitian quadrature operators, $\hat{\gamma}_{(i)}$, as:

$$
\begin{aligned}
& \hat{\gamma}_{(1)}=\left(\hat{\boldsymbol{a}}+\hat{\boldsymbol{a}}^{\dagger}\right) \\
& \hat{\gamma}_{(2)}=-\mathrm{i}\left(\hat{\boldsymbol{a}}-\hat{\boldsymbol{a}}^{\dagger}\right),
\end{aligned}
$$

which implies that the $\hat{\gamma}$ variables have the following anti-commutation relation [3, 10, 11.:

$$
\left\{\hat{\gamma}_{i}, \hat{\gamma}_{j}\right\}=2 \delta_{i j}
$$

The relationship can be inverted, and the extended ladder operators can be expressed as:

$$
\underline{\underline{a}}=\underline{\underline{U}}^{-1} \hat{\gamma} .
$$

Using the transformation $\underline{\underline{U_{0}}}$ it is possible to write an un-normalized Gaussian operator as:

$$
\hat{\Lambda}^{u}(\underline{\underline{Y}})=: \exp \left[\frac{\mathrm{i}}{2} \hat{\gamma}^{T} \underline{\underline{Y}} \hat{\underline{\gamma}}\right]:
$$

We will call the above operators the un-normalized Majorana Gaussian operators. Here we have defined:

$$
\underline{\underline{Y}}=\frac{\mathrm{i}}{2}{\underline{\underline{U_{0}}}}_{\underline{\underline{U_{0}}}}^{-1}
$$

Provided that $\underline{\underline{\mu}}$ has the class D symmetry properties identified above, the matrix $\underline{\underline{Y}}$ is a real antisymmetric matrix. In the single mode case, one can define $Y \equiv Y_{12}$, and since all normally ordered powers beyond first order will vanish,

$$
\hat{\Lambda}^{u}(Y)=1+i Y: \hat{\gamma}_{1} \hat{\gamma}_{2}:=1+2 \hat{n} Y .
$$




\subsection{Unit trace Gaussian operators}

We would like to express the unit trace Gaussian operators in terms of Majorana operators, together with a matrix $\underline{\underline{X}}$ that plays the role of a Majorana covariance. Covariance matrices and Majorana variables have been used in order to study topological edge states correlations in free and interacting fermion systems [64]. They have also been used to study pairing in fermionic systems [62].

For this purpose, we define the following real antisymmetric matrices:

$$
\underline{\underline{X}}=\underline{\underline{U_{0}}}\left[\underline{\underline{J}}-2 \underline{\underline{\sigma}} \underline{\underline{U_{0}}}{ }^{-1}\right.
$$

and an antisymmetric real 'identity-like' matrix

$$
\underline{\underline{I}}=\underline{\underline{\mathrm{U}}} \underline{\underline{\underline{J}}} \underline{\underline{U_{0}}}{ }^{-1}
$$

which can be written explicitly as;

$$
\underline{\underline{\underline{I}}}=\left[\begin{array}{cc}
0 & \mathbf{I} \\
-\mathbf{I} & 0
\end{array}\right] \text {. }
$$

Equation (5) gives a relationship between the variance matrix $\underline{\underline{\sigma}}$ and the $\underline{\underline{\mu}}$ matrix. In terms of the corresponding antisymmetric matrices $\underline{\underline{X}}$ and $\underline{\underline{Y}}$, this relationship is given by:

$$
\underline{\underline{X}}=\underline{\underline{I}}+(\underline{\underline{Y}}+\underline{\underline{I}})^{-1}
$$

In the single-mode case, one has that:

$$
\underline{\underline{X}}=\left[\begin{array}{cc}
0 & 1-1 /(1+Y) \\
1 /(1+Y)-1 & 0
\end{array}\right] \text {. }
$$

In order to define the normalized Gaussian operators using Majorana operators, we first consider the normalization factor. Since the determinant is invariant under unitary transformations we can write it as follows:

$$
\sqrt{\operatorname{det}[\underline{\mathrm{i}} \underline{\underline{\sigma}}]}=\frac{1}{2^{M}} \sqrt{\operatorname{det}[\underline{\underline{\mathcal{I}}}-\underline{\underline{X}}]}
$$

Therefore the unit-trace Majorana Gaussian operator in a variance form is:

$$
\hat{\Lambda}(\underline{\underline{X}})=N(\underline{\underline{X}}): \exp \left[-\mathrm{i} \underline{\hat{\gamma}}^{T}\left[\underline{\underline{\mathcal{I}}}-(\underline{\underline{X}}-\underline{\underline{I}})^{-1}\right] \underline{\hat{\gamma}} / 2\right]:
$$

where

$$
N(\underline{\underline{X}})=\frac{1}{2^{M}} \sqrt{\operatorname{det}[\underline{\underline{\mathcal{I}}}-\underline{\underline{X}}]} .
$$

In the single-mode case, with $X=X_{12}$, the normalization factor is:

$$
N(X)=\frac{1}{2(1+Y)}=\frac{1-X}{2}
$$

where the domain boundaries are such that $-1<X<1$, and:

$$
\hat{\Lambda}(X)=\frac{1-X}{2}+\hat{n} X .
$$

We note that there is a particle-hole symmetry, since $\hat{\Lambda}(X)$ is invariant under the transformation $\hat{a} \rightarrow \hat{a}^{\dagger}, \hat{a}^{\dagger} \rightarrow \hat{a}, X \rightarrow-X$. 


\subsection{Classical domains}

After transformation to Majorana form, there are two possible fermionic representations with different phase-spaces. The fermionic P-representation corresponds to the complex antisymmetric matrices in Majorana space, while the Q-representation corresponds to the real antisymmetric matrices.

As we show explicitly below, the natural phase-space of the Majorana Q-function is bounded. This is because the Gaussian basis that is used in the construction of the Qfunction depends on an antisymmetric matrix $\underline{\underline{X}}$, which exists in one of the irreducible bounded symmetric domains of Cartan [65 67]. In the complex case, these symmetric spaces are called the classical domains by Hua [8,68]. The Gaussian basis has deep links to Lie group theory, since we can define a $2 M \times 2 M$ matrix $\underline{\underline{T}}=\exp [\underline{\underline{X}}]$ such that $\underline{\underline{T}}^{T} \underline{\underline{T}}=\underline{\underline{I}}$, where $\underline{\underline{I}}$ is the usual identity matrix. The $\underline{\underline{T}}$ matrices belong to the orthogonal group $O(2 M)$, which is a Lie group. In the case that $\operatorname{det} \underline{\underline{T}}=1$, the group is $S O(2 M)$. The corresponding Lie algebra of this Lie group is isomorphic to the real $2 M \times 2 M$ antisymmetric matrices.

The classical domains [67] are given in terms of complex matrices $\underline{\underline{Z}}$. This leads to a consideration of the four classical domains, which in the classification of Hua 68 are defined as [66, 69, 71]:

(i) The domain $\mathcal{R}_{I}$ of $m \times n$ complex matrices with: $\underline{\underline{I}}^{(m)}-\underline{\underline{Z Z}}^{\dagger}>0$.

(ii) The domain $\mathcal{R}_{I I}$ of $n \times n$ symmetric complex matrices with: $\underline{\underline{I}}-\underline{\underline{Z Z}}{ }^{*}>0$.

(iii) The domain $\mathcal{R}_{I I I}$ of $n \times n$ skew-symmetric (anti-symmetric) complex matrices with: $\underline{\underline{I}}+\underline{\underline{Z Z}}^{*}>0$.

(iv) The domain $\mathcal{R}_{I V}$ of $n$-dimensional vectors $z=\left(z_{1}, z_{2}, \ldots, z_{n}\right)$, where $z_{k}$ are complex numbers, satisfying: $\left|z z^{T}\right|+1-2 z z^{T}>0, \quad\left|z z^{T}\right|<1$.

The most general Majorana Gaussian operators correspond to the complex domain $\mathcal{R}_{I I I}$, for $n=2 M$, which we refer to as $\mathcal{D}_{C}$. The subspace that corresponds to the hermitian Hamiltonians that we use here has a real phase space of $M(2 M-1)$ dimensions, or $\mathcal{D}_{R}$, which is the real subspace of $\mathcal{R}_{I I I}$.

In our notation, the classical domain boundary of the space of the most general complex antisymmetric matrices $\underline{\underline{Z}}$ considered here is given by:

$$
\underline{\underline{R}}=\underline{\underline{I}}+\underline{\underline{Z Z}}{ }^{*}>0 .
$$

Since $\underline{\underline{R}}$ is positive-definite, and $\underline{\underline{Z Z}}{ }^{*}=-\underline{\underline{Z Z}}^{\dagger}$ is negative definite, we deduce that each element of $\underline{\underline{Z}}$ is bounded, which implies that $\sum_{j}\left|Z_{i j}\right|^{2}<1$ and $\left|Z_{i j}\right|<1$. The classical domain boundary of the real antisymmetric matrices $\underline{\underline{X}}$ is the same, except for the additional requirement that the elements are real.

This restriction of the domain $\mathcal{R}_{I I I}$ corresponds to the hermitian Cartan symmetric space DIII [65,66]. Altland and Zirnbauer [44 classified non-standard symmetry classes for fermionic systems. Here the classification is made by considering the Lie algebra which the symmetry class belongs to [44,72]. In this notation, their symmetry class 
$D$ corresponds to $D_{N} \equiv s o(2 N)$ [44, 70, 72]. The Lie algebra is isomorphic to the real antisymmetric $2 N \times 2 N$ matrices.

The boundary condition can also be written in the real case as:

$$
\underline{\underline{X}}^{+} \underline{\underline{X}}^{-}>0
$$

where we define for convenience,

$$
\underline{\underline{X}}^{ \pm} \equiv \underline{\underline{X}} \pm \underline{\underline{\mathcal{I}}}
$$

For the unordered operator identities derived below, it is simpler to transform the resulting phase-space variable to a modified form such that:

$$
\underline{\underline{x}}=\underline{\underline{\mathcal{I}}}_{\underline{\underline{\mathcal{I}}}}^{\underline{\underline{\mathcal{I}}}}
$$

We also introduce $\underline{\underline{x}}^{ \pm}=\underline{\underline{x}} \pm \underline{\mathrm{i}} \underline{\underline{I}}$, and one can prove that in this case the classical domain bounds are unchanged, with boundaries at:

$$
\underline{\underline{I}}+\underline{\underline{x}}^{2}=\underline{\underline{x}}^{+} \underline{\underline{x}}^{-}>0 \text {. }
$$

\subsection{Resolution of identity}

The definition of a Q-function first requires one to have an expression for the resolution of the Hilbert space identity operator. In order to obtain the most general form of the resolution of identity in the fermion case, we define $\hat{\Lambda}^{N}(\underline{\underline{X}})$ as the following Majorana Gaussian basis:

$$
\hat{\Lambda}^{N}(\underline{\underline{X}})=\frac{1}{\mathcal{N}} \hat{\Lambda}(\underline{\underline{X}}) S\left(\underline{\underline{X^{2}}}\right)
$$

with $\hat{\Lambda}(\underline{\underline{X}})$ given in (18),$S\left(\underline{\underline{X}}^{2}\right)$ is an even, positive scaling function, and $\mathcal{N}$ is a normalization constant that ensures that the identity expansion is normalized. We consider the following form for the scaling factor $S$, which vanishes at the phase-space boundaries:

$$
S=\operatorname{det}\left[\underline{\underline{I}}+\underline{\underline{X}}^{2}\right]^{k / 2}
$$

Following the methods developed earlier [56] for the complex variable Q-function, one can then prove that:

$$
\hat{I}=\int_{\mathcal{D}_{R}} \hat{\Lambda}^{N}(\underline{\underline{X}}) \mathrm{d} X
$$

where the integration is over the bounded real classical domain of antisymmetric matrices, and $d X=\prod_{1 \leq j<k \leq 2 M} d X_{j k}$. The value of the normalization constant is obtained by noting that:

$$
\mathcal{N}=2^{-M} \int_{\mathcal{D}_{R}} S\left(\underline{\underline{X^{2}}}\right) \mathrm{d} X
$$

In the limit that $k \rightarrow 0$, the constant $\mathcal{N}$ is related to the volume of the real classical Hua type III domain [68, 73, 74] using matrix polar coordinate methods, and is given by:

$$
\mathcal{N}=\frac{\pi^{M(M-1 / 2)}}{2^{M}} \prod_{j=1}^{M} \frac{\Gamma(k+j)}{\Gamma\left(k+M+j-\frac{1}{2}\right)}
$$


Thus, for example, in the case of $M=1$, one obtains $\mathcal{N}=1$. We note that these

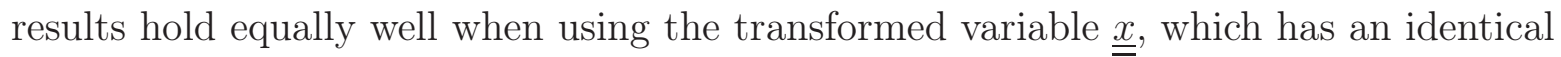
measure, determinant and integration domain. In the single mode case, the two forms are exactly the same.

\section{Majorana differential identities}

The main result of the paper is the derivation of differential identities using Majorana operators and antisymmetric matrices. It is useful to derive differential identities for the normalized Majorana Gaussian operators in terms of the matrices $\underline{\underline{X}}$, because they are related to the covariance matrices $\underline{\underline{\sigma}}$. The fermionic Q-function uses as a basis the Gaussian operators expressed in a symmetric form of these variables. Calculations using the Q-function require differential identities which will involve these variables. Thus, these identities allow one to perform simulations with fermionic phase space representations.

We wish to write differential identities for the action of Majorana operators on the unit-trace Gaussian basis defined in (18). There is a correspondence between Majorana operators and Fermi operators, hence we derive four differential identities which are written using normal, anti-normal ordering and mixed products of normal and antinormal ordering. In order to derive the identities for the normalized Gaussian operators it is convenient to first derive the differential identities for the unnormalized Majorana operators. The next step is to perform a change of variables and use the corresponding normalization factor with its respectively derivative. The differential identities are given below and the details of this procedure are explained in Appendix B, Appendix C and Appendix D.

\subsection{Un-normalized Majorana differential identities}

In order to derive the differential identities for the normalized Gaussian operators we need to derive first the corresponding differential identities for the un-normalized Gaussian operators given in (10). These identities are needed in order to obtain the differential identities in terms of the antisymmetric matrix $\underline{\underline{X}}$. This matrix is related to the covariance matrix $\underline{\underline{\sigma}}$, through the expression given in (13).

We use the convention for matrix derivatives that:

$$
\left[\frac{\mathrm{d}}{\mathrm{d} \underline{\underline{Y}}}\right]_{\mu \nu}=\frac{\mathrm{d}}{\mathrm{d} Y_{\nu \mu}} .
$$

These identities are given in terms of the different forms of the possible orderings between the Majorana variables and the Gaussian operators and are listed below. The corresponding proofs are shown in Appendix B.

- Mixed products:

$$
\left\{\underline{\hat{\underline{\gamma}}}: \underline{\hat{\underline{\gamma}}}^{T} \hat{\Lambda}^{(u)}:\right\}=\mathrm{i}\left[-\underline{\underline{\mathcal{I}}}(2 \underline{\underline{Y}}+\underline{\underline{\mathcal{I}}}) \frac{\mathrm{d}}{\mathrm{d} \underline{\underline{Y}}}+2 \underline{\underline{\mathcal{I}}}\right] \hat{\Lambda}^{(u)} .
$$


The explicit form of $\left\{\underline{\hat{\gamma}}: \underline{\hat{\gamma}}^{T} \hat{\Lambda}:\right\}$, in terms of the creation and annihilation operators is given in Appendix A.

- Normally ordered products:

$$
: \underline{\hat{\gamma} \hat{\gamma}^{T}} \hat{\Lambda}^{(u)}:=\mathrm{i} \frac{d}{d \underline{\underline{Y}}} \hat{\Lambda}^{(u)}
$$

For clarity the detailed expression is given in Appendix A.

- Anti-normally ordered products:

$$
\left\{{\underline{\widehat{\gamma} \hat{\gamma}^{T}}}^{(u)}\right\}=\underline{\mathrm{i}}\left\{(2 \underline{\underline{Y}}+\underline{\underline{I}}) \frac{\mathrm{d}}{\mathrm{d} \underline{\underline{Y}}} \hat{\Lambda}^{u}-2 \hat{\Lambda}^{u}\right\}(2 \underline{\underline{Y}}+\underline{\underline{I}}) \underline{\underline{I}} .
$$

The full expression is given in Appendix A.

\subsection{Normalized Majorana differential identities}

In this section we will give the differential identities for the normalized Gaussian operators given in (19). We note that in order to obtain these identities, we need to perform a change of variables from the matrices $\underline{\underline{Y}}$ to the $\underline{\underline{X}}$ using the relationship given in (15) hence:

$$
\underline{\underline{Y}}=(\underline{\underline{X}}-\underline{\underline{I}})^{-1}-\underline{\underline{I}} \text {. }
$$

We also recall that $\hat{\Lambda}(\underline{\underline{X}})=N(\underline{\underline{X}}) \hat{\Lambda}^{(u)}(\underline{\underline{X}})$, where $N(\underline{\underline{X}})$ is given in (19), The normalized differential identities are given below and in Appendix C we show the procedure in order to obtain these identities.

- Mixed products:

$$
\begin{aligned}
& \left\{\hat{\hat{\gamma}}: \underline{\hat{\gamma}}^{T} \hat{\Lambda}:\right\}=\mathrm{i}\left[\underline{\underline{X}}+\frac{\mathrm{d} \hat{\Lambda}}{\mathrm{d} \underline{\underline{X}}}-\hat{\Lambda}\right] \underline{\underline{X}}^{-} . \\
& : \underline{\hat{\gamma}}\left\{\hat{\hat{\gamma}}^{T} \hat{\Lambda}\right\}:=\mathrm{i}\left[\underline{\underline{X}}^{-} \frac{\mathrm{d} \hat{\Lambda}}{\mathrm{d} \underline{\underline{X}}}-\hat{\Lambda}\right] \underline{\underline{X}}^{+} .
\end{aligned}
$$

- Normally ordered products:

$$
: \underline{\hat{\gamma}}^{T} \hat{\Lambda}:=-\mathrm{i}\left[\underline{\underline{X}}^{-} \frac{\mathrm{d} \hat{\Lambda}}{\mathrm{d} \underline{\underline{X}}}-\hat{\Lambda}\right] \underline{\underline{X}}^{-}
$$

- Anti-normally ordered products:

$$
\left\{\underline{\widehat{\gamma}}^{T} \hat{\Lambda}\right\}=-\mathrm{i}\left[\underline{\underline{X}}+\frac{\mathrm{d} \hat{\Lambda}}{\mathrm{d} \underline{\underline{X}}}-\hat{\Lambda}\right] \underline{\underline{X}}^{+}
$$




\subsection{Unordered Majorana differential identities}

In this section we obtain differential identities that do not use normal or anti-normal ordering as in the previous section. There is no natural normal or anti-normal ordering for Majorana operators, however, the differential identities for the unordered products of the Majorana operators and the Gaussian basis can be obtained by combining the four normalized differential identities given in Section 3.2. The details of the derivation of the differential identities are given in Appendix D. These results are simpler in the

modified variables, $\underline{\underline{x}}=\underline{\underline{\mathcal{I}} X^{T}} \underline{\underline{\mathcal{I}}}$, together with $\underline{\underline{x}}^{ \pm}=\underline{\underline{x}} \pm \mathrm{i} \underline{\underline{I}}$. The resulting identities are:

- Unordered left product:

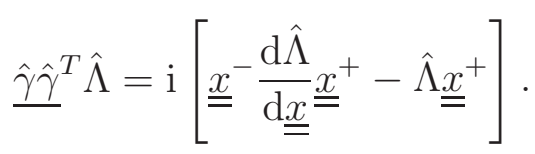

- Unordered right product:

$$
\hat{\Lambda} \underline{\hat{\gamma}}^{T}=\mathrm{i}\left[\underline{\underline{x}}^{+} \frac{\mathrm{d} \hat{\Lambda}}{\mathrm{d} \underline{\underline{x}}} \underline{\underline{x}}^{-}-\hat{\Lambda} \underline{\underline{x}}^{+}\right] .
$$

- Unordered mixed product:

$$
\underline{\hat{\gamma}} \hat{\Lambda} \underline{\hat{\gamma}}^{T}=\mathrm{i}\left[-\underline{\underline{x}}-\frac{\mathrm{d} \hat{\Lambda}}{\mathrm{d} \underline{\underline{x}}} \underline{x}^{-}+\hat{\Lambda} \underline{\underline{x}}^{-}\right] .
$$

\section{Phase space representations}

Phase space representations have been widely used in quantum optics and ultracold atoms. A fermionic P-representation can be introduced using Grassmann variables, which are variables that anti-commute [55], but these are exponentially complex and hence mostly useful for analytic calculations, although they can be used to obtain complex covariance type equations [58].

Another approach is to define fermionic phase space using the Gaussian operators. For fermions there are two known complete representations, which are the fermionic P-representation [36 38, 40, 55] and the fermionic Q-function [43]. In this case the

phase space variables correspond to the matrix of the Gaussian operator, which are the elements of the covariance matrix. Using the Majorana Gaussian representation it is also possible to define phase space representations in terms of the Majorana variables and an antisymmetric matrix. These are the analogous to the fermionic phase space representations.

\subsection{Majorana P-functions}

A Majorana fermionic P-function can be defined in analogy to the fermionic P-function using raising and lowering operators [36 38, 40,55]. In this case the density matrix $\hat{\rho}(t)$ is expanded in terms of the Gaussian basis given in (18). The phase space variables 
are either the antisymmetric matrix $\underline{\underline{X}}$, or its cousin $\underline{\underline{x}}$. Each may be more suitable, depending on whether one wishes to use ordered or unordered Majorana identities. Here we choose the unordered form:

$$
\hat{\rho}(\tau)=\int P(\underline{\underline{x}}, \tau) \hat{\Lambda}(\underline{\underline{x}}) \prod_{i} \mathrm{~d} x_{i} .
$$

The $P(\underline{\underline{x}}, \tau)$ distribution has the same properties as the fermionic $P$-representation:

(i) Normalization: It is normalized to one:

$$
\int_{\mathcal{D}_{C}} P(\underline{\underline{x}}) \prod_{i} \mathrm{~d} x_{i}=1
$$

(ii) Moments: Expectation values of observables $O$ are obtained as:

$$
\langle O\rangle=\int_{\mathcal{D}_{C}} P(\underline{\underline{x}}, \tau) \operatorname{Tr}[O \hat{\Lambda}(\underline{\underline{x}})] \prod_{i} \mathrm{~d} x_{i} \equiv\langle O\rangle_{P} .
$$

\subsection{Majorana Q-functions}

A Majorana Q-function can be defined as:

$$
Q(\underline{\underline{x}})=\operatorname{Tr}\left[\hat{\rho} \hat{\Lambda}^{N}(\underline{\underline{x}})\right] .
$$

This Majorana Q-function has the same properties as the fermionic Q-function which are:

(i) It is defined for any quantum density-matrix.

(ii) It is a positive probability distribution.

(iii) Observables are moments of the distribution. These are obtained as:

$$
\left\langle\hat{O}_{n}\right\rangle=\int_{\mathcal{D}_{R}} Q(\underline{\underline{x}}) O_{n}(\underline{\underline{x}}) \prod_{i} \mathrm{~d} x_{i}=\left\langle\hat{O}_{n}((\underline{\underline{x}}))\right\rangle_{Q} .
$$

\subsection{Single mode Majorana Q-function}

It is instructive to have an expression for the $\mathrm{Q}$ function in the single mode case. Utilizing 18, where there is only one real variable, $x \equiv x_{12}$, and:

$$
\underline{\underline{x}}=\left[\begin{array}{cc}
0 & x \\
-x & 0
\end{array}\right]
$$

We can begin with the expression for the single mode Gaussian operator in Majorana form, which can be re- expressed using the result that:

$$
\widehat{n}=\frac{1+\mathrm{i} \hat{\gamma}_{1} \hat{\gamma}_{2}}{2}
$$

so that

$$
\hat{\Lambda}(x)=\frac{1}{2}+\frac{\mathrm{i}}{2} \hat{\gamma}_{1} \hat{\gamma}_{2} x
$$


All physical density operators are also Gaussian operators in single mode case [36], since if $n \equiv\langle\hat{n}\rangle$, the most general density matrix is

$$
\hat{\rho}=\hat{\Lambda}_{1}(n)=(1-n)|0\rangle\langle 0|+n| 1\rangle\langle 1| .
$$

We arrive at an expression for Majorana Q-function by using the above two equations in 44, to give:

$$
Q(x)=\frac{1}{\mathcal{N}} S(x)\left[\left(\frac{1-x}{2}\right)+n x\right]
$$

where $S(x)=\left[1+x^{2}\right]^{2 p}$.

Just as with the unit trace Gaussian, this has a symmetry between particles and holes, since changing the occupation from $n$ to $1-n$ simply changes the sign of the $\operatorname{argument} x$. We note that in the absence of the normalization factor $S$, the distribution $Q(x)$ would have a discontinuity at the boundary.

\subsection{Observables}

We can obtain observables in terms of the Majorana Q-function using the Majorana differential identities in (45). As an example of this, we consider the Majorana correlation function,

$$
\hat{X}_{\mu \nu} \equiv \frac{\mathrm{i}}{2}\left[\gamma_{\mu}, \gamma_{\nu}\right]
$$

This is a general hermitian observable, which includes the occupation number operators since:

$$
\widehat{n}_{i}=\frac{1+\hat{X}_{i, M+i}}{2}
$$

The expectation is defined as:

$$
\left\langle\hat{X}_{\mu \nu}\right\rangle=\operatorname{Tr}\left[\hat{\rho} \widehat{X}_{\mu v}\right] \text {. }
$$

Next, using the resolution of identity and the unordered product ordering, we can arrive at the expression:

$$
\left\langle\hat{X}_{\mu \nu}\right\rangle=\frac{\mathrm{i}}{2} \int \operatorname{Tr}\left[\widehat{\rho}\left[\widehat{\gamma}_{\mu} \widehat{\gamma}_{v} \hat{\Lambda}^{N}-\widehat{\gamma}_{v} \widehat{\gamma}_{\mu} \hat{\Lambda}^{N}\right]\right] \mathrm{d} \underline{\underline{x}} .
$$

Now we can apply the differential identities corresponding to the unordered product given in (41) obtaining:

$$
\begin{aligned}
\left\langle\widehat{X}_{\mu v}\right\rangle= & \frac{1}{2} \int \operatorname{Tr}\left[\widehat{\rho} \frac{S}{\mathcal{N}}\left[-x_{\mu \alpha}^{-} \frac{\mathrm{d} \hat{\Lambda}}{\mathrm{d} x_{\beta \alpha}} x_{\beta v}^{+}+\hat{\Lambda} x_{\mu v}^{+}\right] \mathrm{d} \underline{=}\right] \\
& -\frac{1}{2} \int \operatorname{Tr}\left[\widehat{\rho} \frac{S}{\mathcal{N}}\left[-x_{v \alpha}^{-} \frac{\mathrm{d} \hat{\Lambda}}{\mathrm{d} x_{\beta \alpha}} x_{\beta \mu}^{+}+\hat{\Lambda} x_{v \mu}^{+}\right] \mathrm{d} \underline{=}\right] .
\end{aligned}
$$


Considering the limit $S \rightarrow 1$ and assuming that the boundary terms vanish and also using the definition of the Q-function, the above equation can be written as:

$$
\left\langle\widehat{X}_{\mu v}\right\rangle=\frac{1}{2} \int\left[-x_{\mu \alpha}^{-} \frac{\mathrm{d}}{\mathrm{d} x_{\beta \alpha}} x_{\beta v}^{+}+x_{\mu v}^{+}\right] Q \mathrm{~d} \underline{\underline{x}}-\frac{1}{2} \int\left[-x_{v \alpha}^{-} \frac{\mathrm{d}}{\mathrm{d} x_{\beta \alpha}} x_{\beta \mu}^{+}+x_{v \mu}^{+}\right] Q \mathrm{~d} \underline{\underline{x}} .
$$

Using the the chain rule the above equation is:

$$
\begin{aligned}
\left\langle\widehat{X}_{\mu v}\right\rangle= & -\frac{1}{2} \int\left[\frac{\mathrm{d}}{\mathrm{d} x_{\beta \alpha}}\left[x_{\mu \alpha}^{-} x_{\beta v}^{+}\right]-2 x_{\mu v}(2 M-1)-x_{\mu v}^{+}\right] Q \mathrm{~d} \underline{\underline{x}} \\
& +\frac{1}{2} \int\left[\frac{\mathrm{d}}{\mathrm{d} x_{\beta \alpha}}\left[x_{v \alpha}^{-} x_{\beta \mu}^{+}\right]-2 x_{v \mu}(2 M-1)-x_{v \mu}^{-}\right] Q \mathrm{~d} \underline{\underline{x}}
\end{aligned}
$$

Here we have assumed that the boundary terms for the normal components of $x_{\mu \alpha}^{-} x_{\beta v}^{+} Q$ vanish at the classical domain boundary, so that the corresponding total derivative integrates to zero:

$$
\int \frac{\mathrm{d}}{\mathrm{d} x_{\beta \alpha}}\left[x_{\mu \alpha}^{-} x_{\beta v}^{+} Q\right] \mathrm{d} \underline{\underline{x}}=0 .
$$

This will leads to the required observable equation:

$$
\langle\underline{\underline{\hat{X}}}\rangle=(4 M-1) \int \underline{\underline{x}} Q(\underline{\underline{x}}) \mathrm{d} \underline{\underline{x}} .
$$

Similarly, we can extend this method to evaluate higher order correlations as well. The assumption of vanishing boundary terms is an important restriction on our results, and would need to be checked in individual cases.

\section{Hamiltonian and time evolution}

Majorana variables have been used to study decoherence effects in Fermi systems [75] or fermionic entanglement 63, 76]. These effects are related to the dynamical evolution of the systems. The Majorana differential identities are useful in order to perform dynamical simulations. In order to illustrate this, we will consider the simplest case of quadratic Hamiltonians. While higher order terms can be treated, these result in diffusion-type differential operators, which are outside the scope of the present article.

\subsection{Quadratic Hamiltonians}

The symmetries of fermionic covariance matrices and quadratic Hamiltonians have identical properties. The general hermitian quadratic Hamiltonian has been widely investigated, and has the form:

$$
\hat{H}=\frac{1}{2}\left[\hat{\boldsymbol{a}}^{\dagger} \mathbf{h} \hat{\boldsymbol{a}}-\hat{\boldsymbol{a}} \boldsymbol{h}^{T} \hat{\boldsymbol{a}}^{\dagger}+\hat{\boldsymbol{a}}^{\dagger} \boldsymbol{\Delta} \hat{\boldsymbol{a}}^{\dagger}-\hat{\boldsymbol{a}} \boldsymbol{\Delta}^{*} \hat{\boldsymbol{a}}\right] .
$$

This Hamiltonian is also known as the Bogoliubov- de Gennes Hamiltonian, which can be written in matrix form using the extended ladder operators as:

$$
\hat{H}=\frac{1}{2} \underline{\hat{a}}^{\dagger} \underline{\underline{H}} \underline{\hat{a}},
$$


where the matrix $\underline{\underline{H}}$ is defined as:

$$
\underline{\underline{H}}=\left(\begin{array}{cc}
\mathbf{h} & \Delta \\
-\Delta^{*} & -\mathbf{h}^{T}
\end{array}\right)
$$

with $\mathbf{h}=\mathbf{h}^{\dagger}$ and $\boldsymbol{\Delta}=-\boldsymbol{\Delta}^{T}$ as the only symmetry restrictions. Altland and Zirnbauer [44] consider this Hamiltonian in order to define the non-standard symmetry classes. This is done by considering how imposing time-reversal and/or spin-rotation symmetry on the Hamiltonian leads to different types of symmetry classes. Each one of these corresponds to a particular type of Lie group that has a corresponding mathematical symmetric space.

Here we will focus on class D symmetry, which has neither time-reversal nor spinrotation invariance, allowing us to treat an arbitrary Fermi system, which means that $\underline{\underline{H}}=\underline{\underline{H}}^{\dagger}$. Using the relationship for the ladder operators and the Majorana operators given in (9) we can express the Hamiltonian of (61) in the following form [2, 77]:

$$
\hat{H}=\frac{i \hbar}{2} \underline{\hat{\gamma}}^{T} \underline{\underline{\Omega} \hat{\gamma}}=\frac{\hbar}{2} \Omega_{\mu \nu} \hat{X}_{\mu \nu}
$$

The expression of the matrix $\underline{\underline{\Omega}}$ in terms of matrices $\mathbf{h}$ and $\Delta$ is:

$$
\underline{\underline{\Omega}}=\frac{1}{2 \mathrm{i} \hbar}\left(\begin{array}{cc}
\mathbf{h}_{-}+\boldsymbol{\Delta}_{-} & i \mathbf{h}_{+}-i \boldsymbol{\Delta}_{+} \\
-i \mathbf{h}_{+}-i \boldsymbol{\Delta}_{+} & \mathbf{h}_{-}-\boldsymbol{\Delta}_{-}
\end{array}\right) .
$$

Here we have defined $\mathbf{h}_{ \pm}=\mathbf{h} \pm \mathbf{h}^{T}$ and $\boldsymbol{\Delta}_{ \pm}=\boldsymbol{\Delta} \pm \boldsymbol{\Delta}^{*}$. We note that $\underline{\underline{\Omega}}$ is a real anti-symmetric matrix: $\underline{\underline{\Omega}}^{T}=-\underline{\underline{\Omega}}$, and therefore satisfies the group properties of these matrices, with a well-defined Haar measure.

\subsection{Time evolution}

The time evolution equation of the Majorana Q-function is obtained by considering that:

$$
\frac{\mathrm{d} Q(\underline{\underline{x}})}{\mathrm{d} t}=\operatorname{Tr}\left[\frac{\mathrm{d} \hat{\rho}}{\mathrm{d} t} \hat{\Lambda}^{N}(\underline{\underline{x}})\right] .
$$

Here we have used the definition of the Majorana Q-function given in (44). Next we consider the time-evolution equation for the density operator:

$$
i \hbar \frac{\partial}{\partial t} \hat{\rho}=[\hat{H}, \hat{\rho}]
$$

On substituting this on the time-evolution equation for the Majorana Q-function and using the cyclic properties of the trace, we get:

$$
\frac{\mathrm{d} Q(\underline{\underline{x}})}{\mathrm{d} t}=\frac{1}{\mathrm{i} \hbar} \operatorname{Tr}\left[\left[\hat{\Lambda}^{N}(\underline{\underline{x}}), \hat{H}\right] \hat{\rho}\right] .
$$

Here we will consider the general Hamiltonian $\hat{H}$ given in (63). Therefore we get that the time-evolution equation for the Majorana Q-function is:

$$
\frac{\mathrm{d} Q(\underline{\underline{x}})}{\mathrm{d} t}=\frac{1}{2 \mathrm{i}} \operatorname{Tr}\left[\hat{\Lambda}^{N} \Omega_{\mu \nu} \hat{X}_{\mu \nu} \hat{\rho}-\Omega_{\mu \nu} \hat{X}_{\mu \nu} \hat{\Lambda}^{N} \hat{\rho}\right] .
$$


We now use the definition of $\underline{\underline{\hat{X}}}$ given in (51), obtaining:

$$
\frac{\mathrm{d} Q(\underline{\underline{x}})}{\mathrm{d} t}=-\frac{1}{4} \operatorname{Tr}\left[\Omega_{\mu \nu}\left[\gamma_{\mu} \gamma_{\nu}-\gamma_{\nu} \gamma_{\mu}, \hat{\Lambda}^{N}\right] \hat{\rho}\right] .
$$
below:

The differential identity for $\left[\gamma_{\mu} \gamma_{\nu}-\gamma_{\nu} \gamma_{\mu}, \widehat{\Lambda}\right]$ is derived from (41). This is given

$$
\left[\gamma_{\mu} \gamma_{\nu}-\gamma_{\nu} \gamma_{\mu}, \widehat{\Lambda}\right]=4\left[x_{\kappa v} \frac{\mathrm{d} \hat{\Lambda}}{\mathrm{d} x_{\kappa \mu}}-x_{\mu \kappa} \frac{\mathrm{d} \hat{\Lambda}}{\mathrm{d} x_{v \kappa}}\right] .
$$

On substituting (69) in (68), we get:

$$
\frac{\mathrm{d} Q(\underline{\underline{X}})}{\mathrm{d} t}=-\operatorname{Tr}\left[\Omega_{\mu \nu} \frac{S}{\mathcal{N}}\left[-x_{\mu \kappa} \frac{\mathrm{d} \hat{\Lambda}}{\mathrm{d} x_{v \kappa}}+\frac{\mathrm{d} \hat{\Lambda}}{\mathrm{d} x_{\kappa \mu}} x_{\kappa v}\right] \hat{\rho}\right] .
$$

Following the steps outlined in Appendix E, we get:

$$
\frac{\mathrm{d} Q(\underline{\underline{X}})}{\mathrm{d} t}=-\Omega_{\mu \nu}\left[-x_{\mu \kappa} \frac{\mathrm{d} Q}{\mathrm{~d} x_{v \kappa}}+\frac{\mathrm{d} Q}{\mathrm{~d} x_{\kappa \mu}} x_{\kappa v}\right]
$$

Next, using the product rule, (71) can be written as:

$$
\frac{\mathrm{d} Q(\underline{\underline{X}})}{\mathrm{d} t}=\Omega_{\mu \nu}\left[\frac{\mathrm{d}}{\mathrm{d} x_{v \kappa}}\left(x_{\mu \kappa} Q\right)-\frac{\mathrm{d}}{\mathrm{d} x_{\kappa \mu}}\left(x_{\kappa v} Q\right)\right] .
$$

The method of characteristics allows us to solve the above equation as:

$$
\frac{d \underline{\underline{x}}}{d t}=[\underline{\underline{\Omega}}, \underline{\underline{x}}] \text {. }
$$

We note that the Heisenberg equations of motion for the Majorana operators in this case are identical, and are given by:

$$
\frac{\mathrm{d} \underline{\underline{\underline{X}}}(t)}{\mathrm{d} t}=[\underline{\underline{\Omega}}, \underline{\underline{\hat{X}}}] \text {. }
$$

This result is valid for completely arbitrary quadratic real antisymmetric matrices $\underline{\underline{\Omega}}$, even with anomalous terms or complex frequency matrices. Since the operator equations for $\underline{\underline{X}}$ are simply proportional to the mean value equations, which in turn are proportional to the characteristic equations for $\underline{\underline{x}}$, this provides a verification of the phase-space results. The utility of the Q-function method in this case is that the general Q-function can be computed given an arbitrary initial distribution. One is not restricted to just calculating mean values of the lowest order correlation function.

\subsection{Bosonic Q-function}

For comparison purposes, we can also write a time evolution for the bosonic Q-function [78] in a similar form that the one described in the above section for fermions. In this case we consider the following Hamiltonian of a non-interacting Bose gas:

$$
\hat{H}=\hbar \hat{\boldsymbol{a}}^{\dagger} \boldsymbol{\omega} \hat{\boldsymbol{a}}
$$


Following the standard techniques for phase-space representations, where one uses the corresponding identities that map phase space variables into c-numbers [79], we obtain the following time evolution equation for the bosonic $\mathrm{Q}$ function, $Q_{B}$ :

$$
\frac{d Q_{B}(\boldsymbol{\alpha})}{d t}=i \boldsymbol{\omega}\left[\frac{\partial}{\partial \boldsymbol{\alpha}} \boldsymbol{\alpha}-\frac{\partial}{\partial \boldsymbol{\alpha}^{*}} \boldsymbol{\alpha}^{*}\right] Q_{B} .
$$

We use the methods of characteristics in order to solve this differential equation obtaining:

$$
\frac{d \boldsymbol{\alpha}}{d t}=-i \boldsymbol{\omega} \boldsymbol{\alpha}
$$

Next, we define the following two real vectors:

$$
\begin{aligned}
& \boldsymbol{\alpha}_{x}=\frac{1}{2}\left[\boldsymbol{\alpha}+\boldsymbol{\alpha}^{*}\right], \\
& \boldsymbol{\alpha}_{y}=\frac{1}{2 i}\left[\boldsymbol{\alpha}-\boldsymbol{\alpha}^{*}\right] .
\end{aligned}
$$

Consequently we can introduce the following real vector:

$$
\underline{\boldsymbol{\alpha}}=\left[\begin{array}{c}
\boldsymbol{\alpha}_{x} \\
\boldsymbol{\alpha}_{y}
\end{array}\right]
$$

and a matrix of quadrature correlations, analogous to the Majorana phase space matrix, where

$$
\underline{x}_{b}=\underline{x x}^{T} .
$$

Using these expressions in (77), leads to a time evolution equation for $\underline{x}_{b}$ of the form:

$$
\frac{d}{d t}\left(\underline{\underline{x}}_{b}\right)=\left[\underline{\underline{\Omega}}_{b}, \underline{\underline{x}}_{b}\right]
$$

where $\underline{\underline{\Omega}}_{b}=\left(\begin{array}{cc}0 & \omega \\ -\omega & 0\end{array}\right)$.

In summary, for the usual bosonic case, one also obtains a simple characteristic evolution, provided the Hamiltonian has no anomalous terms. More generally, the results are more complicated, and do not follow a simple first-order equation. In the Fermi Q-function case, a first-order characteristic evolution is obtained for any quadratic Hamiltonian.

\section{Time-Evolution of open quantum systems}

We now consider the following application of our method: the time evolution of an open quantum system. This will include the interaction of a system with the environment, which will show that our method can treat dissipative systems as well. The system is a small quantum dot coupled to a zero temperature reservoir. Here it is convenient to use the ordered identities, as the dissipative master-equation terms have normal ordering. 


\subsection{Master Equation}

The time evolution of the density operator of this model is given by a master equation [37]:

$$
\frac{d \hat{\rho}}{d t}=-i \omega \hat{a}^{\dagger} \hat{a} \hat{\rho}+i \omega \hat{\rho} \hat{a}^{\dagger} \hat{a}+\gamma\left(\hat{a} \hat{\rho} \hat{a}^{\dagger}-\frac{1}{2} \hat{a}^{\dagger} \hat{a} \hat{\rho}-\frac{1}{2} \hat{\rho} \hat{a}^{\dagger} \hat{a}\right) .
$$

In the case of a multi-mode quantum system we get

$$
\frac{d \hat{\rho}}{d t}=-i \omega_{j i} \hat{a}_{i}^{\dagger} \hat{a}_{j} \hat{\rho}+i \omega_{j i} \hat{\rho} \hat{a}_{i}^{\dagger} \hat{a}_{j}+\gamma_{i j}\left(\hat{a}_{i} \hat{\rho} \hat{a}_{j}^{\dagger}-\frac{1}{2} \hat{a}_{j}^{\dagger} \hat{a}_{i} \hat{\rho}-\frac{1}{2} \hat{\rho} \hat{a}_{j}^{\dagger} \hat{a}_{i}\right)
$$

provided $\boldsymbol{\omega}=\boldsymbol{\omega}^{T}$ and $\boldsymbol{\gamma}=\boldsymbol{\gamma}^{T}$. On substituting this equation for the time evolution of the Q-function given in (65) we obtain:

$$
\begin{aligned}
\frac{d Q}{d t}= & i \operatorname{Tr}\left[\omega_{j i}\left[\widehat{a}_{i}^{\dagger} \widehat{a}_{j}, \hat{\Lambda}^{N}(\underline{\underline{X}})\right] \widehat{\rho}\right]+\operatorname{Tr}\left[\hat{\Lambda}^{N}(\underline{\underline{X}}) \gamma_{i j} \widehat{a}_{i} \widehat{\rho} \widehat{a}_{j}^{\dagger}\right] \\
& -\frac{1}{2} \operatorname{Tr}\left[\gamma_{i j}\left[\widehat{a}_{i}^{\dagger} \widehat{a}_{j}, \hat{\Lambda}^{N}(\underline{\underline{X}})\right]_{+} \widehat{\rho}\right] .
\end{aligned}
$$

We now wish to express the above results in terms of the Majorana differential identities given in Section 3. First, using the expressions of the different orderings of the Majorana variables and the Gaussian operators given in Appendix C we rewrite (82) as:

$$
\begin{aligned}
\frac{d Q}{d t}= & -\frac{i}{2} \frac{1}{\mathcal{N}} S \operatorname{Tr}\left[\tilde{\Omega}_{\kappa \nu}\left\{\underline{\hat{\gamma}}: \underline{\hat{\gamma}}^{T} \hat{\Lambda}:\right\}_{\nu \kappa} \hat{\rho}\right] \\
& -\frac{1}{2 i} \frac{1}{\mathcal{N}} S \operatorname{Tr}\left[\Upsilon_{\kappa \nu}\left(: \underline{\hat{\gamma}} \hat{\gamma}^{T} \hat{\Lambda}:{ }_{\nu \kappa}-\left\{\underline{\hat{\gamma}}: \underline{\hat{\gamma}}^{T} \hat{\Lambda}:\right\}_{\nu \kappa}\right) \hat{\rho}\right]-\gamma_{i j} \delta_{i j} Q .
\end{aligned}
$$

Here we have defined

$$
\begin{aligned}
& \underline{\tilde{\Omega}}=\left(\begin{array}{cc}
\omega & 0 \\
0 & \omega
\end{array}\right), \quad \text { and } \\
& \underline{\underline{\Upsilon}}=\left(\begin{array}{cc}
0 & -\frac{\gamma}{2} \\
\frac{\gamma}{2} & 0
\end{array}\right) .
\end{aligned}
$$

On using the Majorana differential identities given in (37) and (39) we get that the time evolution equation is:

$$
\begin{aligned}
\frac{d Q}{d t}= & \frac{1}{2} \tilde{\Omega}_{\kappa \nu} \frac{d}{d X_{p \ell}}\left(X_{\nu \ell}^{+} Q X_{p \kappa}^{-}\right)+\Upsilon_{\kappa \nu} \frac{d}{d X_{p l}}\left(X_{\nu \ell} Q X_{p \kappa}^{-}\right) \\
& -(4 M-1) X_{\nu \kappa} \Upsilon_{\kappa \nu} Q+(2 M-1) \Upsilon_{\kappa \nu} \mathcal{I}_{\nu \kappa} .
\end{aligned}
$$

Details of the calculations are given in Appendix F. We will next illustrate the dynamic behavior for this dissipative system for the single mode case. 


\subsection{Single mode case}

We wish to show the dynamic behavior of the open quantum system. In order to do this, we will study the nature of trajectories for the single mode case, where $\underline{\underline{X}}=\underline{\underline{x}}$. In this case, defining $X=X_{12}$, (86) reduces to:

$$
\frac{d Q}{d t}=\gamma\left[\frac{d}{d X}[Q X(X-1)]\right]+\gamma(1-3 X) Q
$$

We can solve the above differential equation using method of characteristics which leads to:

$$
\frac{d X}{d t}=\gamma X(1-X)
$$

On integrating this equation we get:

$$
t-t_{0}=\ln \left[\frac{X}{1-X}\right] .
$$

This can be written as:

$$
e^{-\left(t-t_{0}\right)}=\frac{1}{X}-1
$$

which gives two possible solutions:

$$
X=\frac{1}{1 \pm e^{-\left(t-t_{0}\right)}} .
$$

In order to get this result we have used that since $t_{0}$ is arbitrary, it can be chosen as complex, so for $X<0$, one has: $t_{0} \rightarrow i \pi+t_{0}$, which gives the minus sign. Therefore, if $t \rightarrow \infty$, then, either with the plus sign, $X(0)>0$, leads to $X \rightarrow 1$ or with the minus sign, $X(0)<0$, leads to $X \rightarrow-\infty$. This means that for $X(0)<0$ the trajectory crosses the boundary after a finite time, and is lost. The solution of the differential equation (87) is depicted graphically in figure 1.

One usually has a first order Fokker Plank equation when dealing with a damped P-function for bosons. It decays down to a delta function. This means that for the bosonic $\mathrm{P}$ function, every trajectory decays to zero. Then the long-time solution in that case is a delta function with zero width. However, for bosons, the Q-function method would include a diffusion term, and give a finite width.

In our case we have a first-order differential equation for a fermionic $\mathrm{Q}$ function. In this case we get a finite width for the ground state solution for the single mode case. We would normally expect to obtain this through a diffusion term in the Fokker Planck equation. However we have no diffusion term in our calculation, but we still get a finite width, which makes the dynamics unusual.

In this case, the Q-function dissipative dynamics has both sources and sinks, with weighted trajectories being generated at the origin, and being lost at the boundary at $X=-1$. 


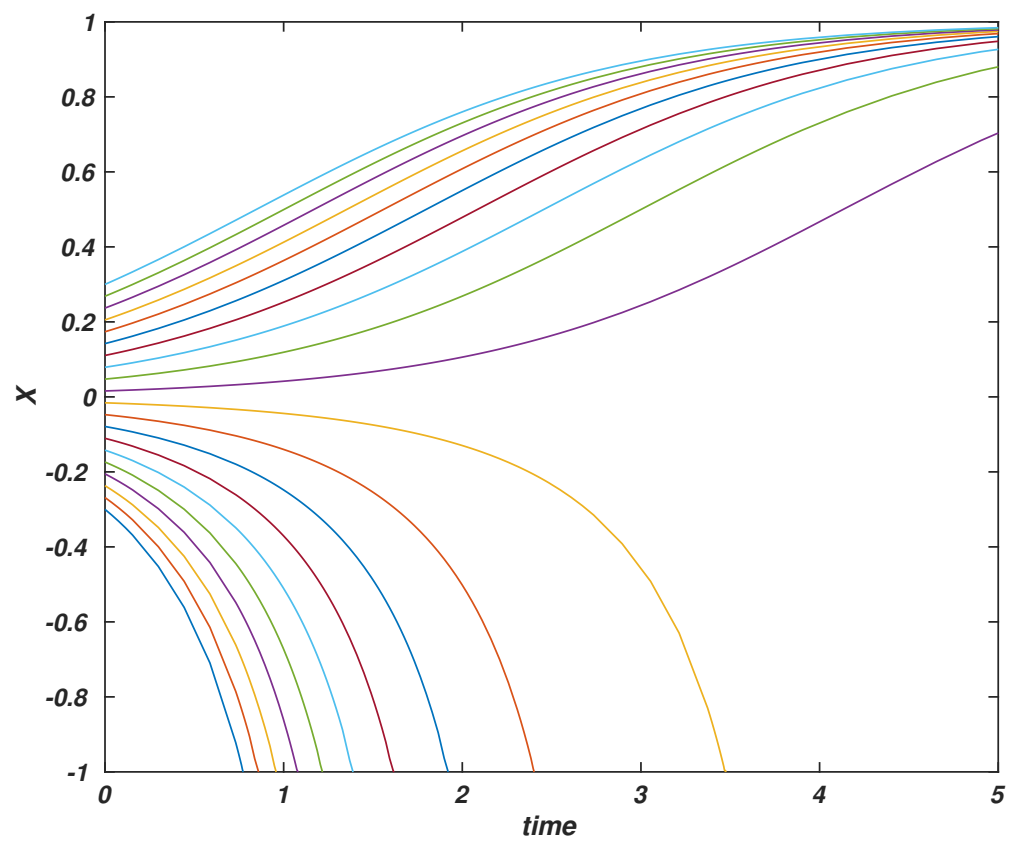

Figure 1. Dynamics of a single mode quantum dot coupled to a zero temperature reservoir.

\section{Summary}

In summary, we have introduced a formalism for a fermionic phase space representation in terms of Majorana operators and a phase-space of antisymmetric matrices defined within a Cartan bounded homogeneous domain. This phase space representation uses as a basis the Majorana Gaussian operators, whose symmetry group is associated with the same classical domain of real antisymmetric matrices.

We have derived differential identities for the Majorana Gaussian operators. These identities are relevant to the important tasks of computing observables and carrying out dynamical simulations. As a simple illustration, we have derived time evolution equations of the Majorana Q-function for the number conserving Hamiltonian, in the case of a general quadratic Hamiltonian.

We have also obtained time evolution equations for a quantum dot coupled to a zero temperature reservoir, which is an example of a dissipative system. The results obtained are quite different to those in the bosonic Q-function representation of Husimi. For example, we can treat all quadratic Hamiltonians as simple trajectory evolution not just those with number-conserving Hamiltonians.

These results show the usefulness of the Majorana differential identities. As an example, our results can also be used to study the dynamics of shock-wave formation in a one-dimensional Fermi gas at finite temperature. This study will be carried out elsewhere. 
Phase space methods for Majorana fermions

\section{Acknowledgments}

We acknowledge the support of the Australian Research Council.

\section{Appendix A. Explicit form of the different orderings for the Majorana variables and Gaussian operators}

In this section we write the explicit forms of the possible different orderings of the Majorana variables with the Majorana Gaussian operators. These expressions are given following the order in which the differential identities were given.

Appendix A.1. First mixed product

$$
\begin{aligned}
& \left\{\underline{\hat{\gamma}}: \underline{\hat{\gamma}}^{T} \hat{\Lambda}:\right\}= \\
& \left(\begin{array}{cc}
\boldsymbol{T}_{1}-\left(\hat{\Lambda} \hat{\boldsymbol{a}} \hat{\boldsymbol{a}}^{\dagger}\right)^{T}-\left(\hat{\boldsymbol{a}}^{\dagger T} \hat{\Lambda} \hat{\boldsymbol{a}}^{\dagger}\right)^{T}-\mathrm{i}\left[\boldsymbol{T}_{2}-\left(\hat{\Lambda} \hat{\boldsymbol{a}} \hat{\boldsymbol{a}}^{\dagger}\right)^{T}+\left(\hat{\boldsymbol{a}} \dagger T \hat{\Lambda} \hat{\boldsymbol{a}}^{\dagger}\right)^{T}\right] \\
-\mathrm{i}\left[\boldsymbol{T}_{1}+\left(\hat{\Lambda} \hat{\boldsymbol{a}} \hat{\boldsymbol{a}}^{\dagger}\right)^{T}+\left(\hat{\boldsymbol{a}}^{\dagger T} \hat{\Lambda} \hat{\boldsymbol{a}}^{\dagger}\right)^{T}\right]-\left[\boldsymbol{T}_{2}+\left(\hat{\boldsymbol{a}}{ }^{\dagger T} \hat{\Lambda} \hat{\boldsymbol{a}}^{\dagger}\right)^{T}-\left(\hat{\Lambda} \hat{\boldsymbol{a}} \hat{\boldsymbol{a}}^{\dagger}\right)^{T}\right]
\end{array}\right),
\end{aligned}
$$

where $\boldsymbol{T}_{1}=\hat{\boldsymbol{a}} \hat{\Lambda} \hat{\boldsymbol{a}}^{T}+\hat{\boldsymbol{a}} \hat{\boldsymbol{a}}^{\dagger} \hat{\Lambda}$ and $\boldsymbol{T}_{2}=\hat{\boldsymbol{a}} \hat{\Lambda} \hat{\boldsymbol{a}}^{T}-\hat{\boldsymbol{a}} \hat{\boldsymbol{a}}^{\dagger} \hat{\Lambda}$.

Appendix A.2. Second mixed product

$$
: \hat{\gamma}\{\hat{\gamma} \hat{\Lambda}\}:=\left(\begin{array}{cc}
\boldsymbol{T}_{1}^{\prime}-\left(\hat{\Lambda} \hat{\boldsymbol{a}}^{\dagger T} \hat{\boldsymbol{a}}^{T}\right)^{T}-\left(\hat{\boldsymbol{a}} \hat{\Lambda} \hat{\boldsymbol{a}}^{T}\right)^{T} & \mathrm{i}\left[\boldsymbol{T}_{2}^{\prime}-\hat{\boldsymbol{a}}^{\dagger T} \hat{\boldsymbol{a}}^{T} \hat{\Lambda}+\hat{\boldsymbol{a}}^{\dagger T} \hat{\Lambda} \hat{\boldsymbol{a}}^{\dagger}\right] \\
\mathrm{i}\left[\boldsymbol{T}_{1}^{\prime}+\left(\hat{\Lambda} \hat{\boldsymbol{a}}^{\dagger T} \hat{\boldsymbol{a}}^{T}\right)^{T}+\left(\hat{\boldsymbol{a}} \hat{\Lambda} \hat{\boldsymbol{a}}^{T}\right)^{T}\right] & \boldsymbol{T}_{2}^{\prime}+\hat{\boldsymbol{a}}^{\dagger T} \hat{\boldsymbol{a}}^{T} \hat{\Lambda}-\hat{\boldsymbol{a}}^{\dagger T} \hat{\Lambda} \hat{\boldsymbol{a}}^{\dagger}
\end{array}\right)
$$

where $\boldsymbol{T}_{1}^{\prime}=+\hat{\boldsymbol{a}}^{\dagger T} \hat{\boldsymbol{a}}^{T} \hat{\Lambda}+\hat{\boldsymbol{a}}^{\dagger T} \hat{\Lambda} \hat{\boldsymbol{a}}^{\dagger}$ and $\boldsymbol{T}_{2}^{\prime}=-\left(\hat{\Lambda} \hat{\boldsymbol{a}}^{\dagger T} \hat{\boldsymbol{a}}^{T}\right)^{T}+\left(\hat{\boldsymbol{a}} \hat{\Lambda} \hat{\boldsymbol{a}}^{T}\right)^{T}$.

Appendix A.3. Normally ordered products

$$
: \underline{\hat{\gamma} \hat{\gamma}^{T}} \hat{\Lambda}:=\left(\begin{array}{cc}
\boldsymbol{T}_{3}+\hat{\boldsymbol{a}}^{\dagger T} \hat{\Lambda} \hat{\boldsymbol{a}}^{T}+\hat{\boldsymbol{a}}^{\dagger T} \hat{\boldsymbol{a}}^{\dagger} \hat{\Lambda} & -\mathrm{i}\left[\boldsymbol{T}_{4}+\hat{\boldsymbol{a}}^{\dagger T} \hat{\Lambda} \hat{\boldsymbol{a}}^{T}-\hat{\boldsymbol{a}}^{\dagger T} \hat{\boldsymbol{a}}^{\dagger} \hat{\Lambda}\right] \\
-\mathrm{i}\left[\boldsymbol{T}_{3}-\hat{\boldsymbol{a}}^{\dagger T} \hat{\Lambda} \hat{\boldsymbol{a}}^{T}-\hat{\boldsymbol{a}}^{\dagger T} \hat{\boldsymbol{a}}^{\dagger} \hat{\Lambda}\right] & -\left[\boldsymbol{T}_{4}-\hat{\boldsymbol{a}}^{\dagger T} \hat{\Lambda} \hat{\boldsymbol{a}}^{T}+\hat{\boldsymbol{a}}^{\dagger T} \hat{\boldsymbol{a}}^{\dagger} \hat{\Lambda}\right]
\end{array}\right),
$$

where $\boldsymbol{T}_{3}=\hat{\Lambda} \hat{\boldsymbol{a}} \hat{\boldsymbol{a}}^{T}-\left(\hat{\boldsymbol{a}}^{\dagger T} \hat{\Lambda} \hat{\boldsymbol{a}}^{T}\right)^{T}$ and $\boldsymbol{T}_{4}=\hat{\Lambda} \hat{\boldsymbol{a}} \hat{\boldsymbol{a}}^{T}+\left(\hat{\boldsymbol{a}}^{\dagger T} \hat{\Lambda} \hat{\boldsymbol{a}}^{T}\right)^{T}$.

Appendix A.4. Anti-normally ordered products

$$
\left\{\underline{\hat{\gamma} \hat{\gamma}^{T} \hat{\Lambda}}\right\}=\left(\begin{array}{cc}
\boldsymbol{T}_{5}-\left(\hat{\boldsymbol{a}} \hat{\Lambda} \hat{\boldsymbol{a}}^{\dagger}\right)^{T}+\hat{\Lambda} \hat{\boldsymbol{a}}^{\dagger T} \hat{\boldsymbol{a}}^{\dagger}-\mathrm{i}\left[\boldsymbol{T}_{6}-\left(\hat{\boldsymbol{a}} \hat{\Lambda} \hat{\boldsymbol{a}}^{\dagger}\right)^{T}-\hat{\Lambda} \hat{\boldsymbol{a}}^{\dagger T} \hat{\boldsymbol{a}}^{\dagger}\right] \\
-\mathrm{i}\left[\boldsymbol{T}_{5}+\left(\hat{\boldsymbol{a}} \hat{\Lambda} \hat{\boldsymbol{a}}^{\dagger}\right)^{T}-\hat{\Lambda} \hat{\boldsymbol{a}}^{\dagger T} \hat{\boldsymbol{a}}^{\dagger}\right]-\left[\boldsymbol{T}_{6}+\left(\hat{\boldsymbol{a}} \hat{\Lambda} \hat{\boldsymbol{a}}^{\dagger}\right)^{T}+\hat{\Lambda} \hat{\boldsymbol{a}}^{\dagger T} \hat{\boldsymbol{a}}^{\dagger}\right]
\end{array}\right),
$$

where $\boldsymbol{T}_{5}=\hat{\boldsymbol{a}} \hat{\boldsymbol{a}}^{T} \hat{\Lambda}+\hat{\boldsymbol{a}} \hat{\Lambda} \hat{\boldsymbol{a}}^{\dagger}$ and $\boldsymbol{T}_{6}=\hat{\boldsymbol{a}} \hat{\boldsymbol{a}}^{T} \hat{\Lambda}-\hat{\boldsymbol{a}} \hat{\Lambda} \hat{\boldsymbol{a}}^{\dagger}$. 
Appendix A.5. Unordered products

$\underline{\hat{\gamma}}^{T} \hat{\Lambda}=\left[\begin{array}{cc}\boldsymbol{T}_{7}+\hat{\boldsymbol{a}}^{\dagger T} \hat{\boldsymbol{a}}^{\dagger} \hat{\Lambda}+\hat{\boldsymbol{a}}^{\dagger T} \hat{\boldsymbol{a}}^{T} \hat{\Lambda} & \mathrm{i}\left[\boldsymbol{T}_{8}+\hat{\boldsymbol{a}}^{\dagger T} \hat{\boldsymbol{a}}^{\dagger} \hat{\Lambda}-\hat{\boldsymbol{a}}^{\dagger T} \hat{\boldsymbol{a}}^{T} \hat{\Lambda}\right] \\ \mathrm{i}\left[-\boldsymbol{T}_{7}+\hat{\boldsymbol{a}}^{\dagger T} \hat{\boldsymbol{a}}^{\dagger} \hat{\Lambda}+\hat{\boldsymbol{a}}^{\dagger T} \hat{\boldsymbol{a}}^{T} \hat{\Lambda}\right] & \boldsymbol{T}_{8}-\hat{\boldsymbol{a}}^{\dagger T} \hat{\boldsymbol{a}}^{\dagger} \hat{\Lambda}+\hat{\boldsymbol{a}}^{\dagger T} \hat{\boldsymbol{a}}^{T} \hat{\Lambda}\end{array}\right]$

where $\boldsymbol{T}_{7}=\hat{\boldsymbol{a}} \hat{\boldsymbol{a}}^{\dagger} \hat{\Lambda}+\hat{\boldsymbol{a}} \hat{\boldsymbol{a}}^{T} \hat{\Lambda}$ and $\boldsymbol{T}_{8}=\hat{\boldsymbol{a}} \hat{\boldsymbol{a}}^{\dagger} \hat{\Lambda}-\hat{\boldsymbol{a}} \hat{\boldsymbol{a}}^{T} \hat{\Lambda}$.

\section{Appendix B. Majorana differential identities for un-normalized ordered products}

In this Section we give the detailed proofs of the un-normalized, ordered product differential identities shown in Section 3. We first give the proof for the un-normalized Majorana differential identities and then in the next section we use these identities in order to obtain the normalized ones.

The following differential identities are given considering the different types of ordering of the Majorana variables and Gaussian operators. The explicit form of these are given in Appendix A. These identities uses the un-normalized Gaussian operators given in (10).

\section{Appendix B.1. Mixed products}

Here we are considering products of the Majorana variables and Gaussian operators of the form $\left\{\hat{\underline{\gamma}}: \underline{\hat{\gamma}}^{T} \hat{\Lambda}^{(u)}:\right\}$. In this case the differential identity is:

$$
\left\{\hat{\hat{\gamma}}: \underline{\hat{\gamma}}^{T} \hat{\Lambda}^{(u)}:\right\}=\mathrm{i}\left[-\underline{\underline{\mathcal{I}}}(2 \underline{\underline{Y}}+\underline{\underline{I}}) \frac{\mathrm{d}}{\mathrm{d} \underline{\underline{Y}}}+2 \underline{\underline{\mathcal{I}}}\right] \hat{\Lambda}^{(u)}
$$

Proof. To prove this identity we proceed by using Grassmann variables and fermionic coherent states. We also use the following result [36] that is already known, but with more details included for clarity and completeness:

$$
\begin{aligned}
\left\{\underline{\underline{a}}: \underline{\underline{a}}^{\dagger} \hat{\Lambda}^{(u)}:\right\} & =\int \mathrm{d} \underline{\gamma} \mathrm{d} \underline{\beta} \mathrm{d} \underline{\alpha} \mathrm{d} \underline{\epsilon}|\gamma\rangle\left\langle\gamma\left|\left\{\underline{\underline{a}}:|\boldsymbol{\beta}\rangle\left\langle\boldsymbol{\beta}\left|\underline{\underline{a}}^{\dagger} \widehat{\Lambda}^{(u)}\right| \boldsymbol{\alpha}\right\rangle\langle\boldsymbol{\alpha}|:\right\}\right| \boldsymbol{\epsilon}\right\rangle\langle\boldsymbol{\epsilon}| \\
& =\int \mathrm{d} \underline{\gamma} \mathrm{d} \underline{\beta} \mathrm{d} \underline{\alpha} \mathrm{d} \underline{\epsilon}|\gamma\rangle\langle\boldsymbol{\epsilon}| \exp \left[-\overline{\boldsymbol{\alpha}} \boldsymbol{\alpha}-\overline{\boldsymbol{\beta}} \boldsymbol{\beta}-\frac{1}{2} \bar{\gamma} \boldsymbol{\gamma}-\frac{1}{2} \overline{\boldsymbol{\epsilon}} \boldsymbol{\epsilon}+\overline{\boldsymbol{\gamma}} \boldsymbol{\beta}+\overline{\boldsymbol{\alpha}} \boldsymbol{\epsilon}\right] \\
& \times\left[\begin{array}{c}
\boldsymbol{\beta} \\
\overline{\boldsymbol{\alpha}}
\end{array}\right]\left[\begin{array}{ll}
\overline{\boldsymbol{\beta}} & \boldsymbol{\alpha}
\end{array}\right] \exp \left[-\frac{1}{2}\left[\begin{array}{ll}
\overline{\boldsymbol{\beta}} & \boldsymbol{\alpha}
\end{array}\right](\underline{\underline{\mu}}+\underline{\underline{J}})\left[\begin{array}{c}
\boldsymbol{\alpha} \\
\overline{\boldsymbol{\beta}}
\end{array}\right]\right] \\
& =\int \mathrm{d} \underline{\mathrm{\gamma}} \underline{\mathrm{\beta}} \mathrm{d} \underline{\alpha} \mathrm{d} \underline{\epsilon}|\boldsymbol{\gamma}\rangle\langle\boldsymbol{\epsilon}| \exp \left[-\overline{\boldsymbol{\alpha}} \boldsymbol{\alpha}-\overline{\boldsymbol{\beta}} \boldsymbol{\beta}-\frac{1}{2} \bar{\gamma} \boldsymbol{\gamma}-\frac{1}{2} \overline{\boldsymbol{\epsilon}} \boldsymbol{\epsilon}+\overline{\boldsymbol{\gamma}} \boldsymbol{\beta}+\overline{\boldsymbol{\alpha}} \boldsymbol{\epsilon}\right] \\
& \left.\times\left[\underline{\underline{J}}(\underline{\underline{\mu}}+\underline{\underline{J}})\left[\begin{array}{c}
\boldsymbol{\alpha} \\
\overline{\boldsymbol{\beta}}
\end{array}\right]\left[\begin{array}{ll}
\overline{\boldsymbol{\beta}} & \boldsymbol{\alpha}
\end{array}\right]-\underline{\underline{J}}\right] \exp \left[\begin{array}{cc}
\overline{\boldsymbol{\beta}} & \boldsymbol{\alpha}
\end{array}\right] \frac{(\underline{\underline{\mu}}+\underline{\underline{J}})}{2}\left[\begin{array}{c}
\boldsymbol{\alpha} \\
\overline{\boldsymbol{\beta}}
\end{array}\right]\right] .
\end{aligned}
$$


Here $|\boldsymbol{\alpha}\rangle,|\boldsymbol{\beta}\rangle,|\boldsymbol{\gamma}\rangle$ and $|\boldsymbol{\epsilon}\rangle$ denote fermionic coherent states, while $\boldsymbol{\alpha}, \boldsymbol{\beta}, \boldsymbol{\gamma}$ and $\boldsymbol{\epsilon}$ are the corresponding Grassmann variables, and $\mathrm{d} \underline{\gamma}, \mathrm{d} \beta, \mathrm{d} \underline{\alpha}$ and $\mathrm{d} \underline{\epsilon}$ are the corresponding $2 M$ integration measures. In order to obtain the identity given in (B.1) we have used an integration by parts and the properties of Grassmann calculus, as well as the eigenvalue properties for the fermionic coherent states in the form $\hat{\boldsymbol{a}}|\boldsymbol{\alpha}\rangle=\boldsymbol{\alpha}|\boldsymbol{\alpha}\rangle$. We have also used the resolution of unity or completeness identity of the fermionic coherent states, together with the inner product property of coherent states which are given below:

$$
\begin{aligned}
\int d \underline{\alpha}|\boldsymbol{\alpha}\rangle\langle\boldsymbol{\alpha}| & =\mathbf{I}, \\
\langle\boldsymbol{\beta} \mid \boldsymbol{\alpha}\rangle & =\exp [\overline{\boldsymbol{\beta}} \boldsymbol{\alpha}-(\overline{\boldsymbol{\beta}} \boldsymbol{\beta}+\overline{\boldsymbol{\alpha}} \boldsymbol{\alpha}) / 2] .
\end{aligned}
$$

One important step is to express the variables $\left[\begin{array}{c}\boldsymbol{\beta} \\ \overline{\boldsymbol{\alpha}}\end{array}\right]$ in the form $\left[\begin{array}{c}\boldsymbol{\alpha} \\ \overline{\boldsymbol{\beta}}\end{array}\right]$, which is the one given in the exponential of the Gaussian operators. This is done by using the following identities, which use the derivative of the Gaussian operator and Grassmann calculus:

$$
\begin{aligned}
& {\left[\begin{array}{c}
\boldsymbol{\beta} \\
\overline{\boldsymbol{\alpha}}
\end{array}\right] \exp [-\overline{\boldsymbol{\alpha}} \boldsymbol{\alpha}-\overline{\boldsymbol{\beta}} \boldsymbol{\beta}]=\left[\begin{array}{c}
-\frac{\partial}{\partial \overline{\bar{\beta}}} \\
\frac{\partial}{\partial \boldsymbol{\alpha}}
\end{array}\right] \exp [-\overline{\boldsymbol{\alpha}} \boldsymbol{\alpha}-\overline{\boldsymbol{\beta}} \boldsymbol{\beta}] .} \\
& \exp [-\overline{\boldsymbol{\alpha}} \boldsymbol{\alpha}-\overline{\boldsymbol{\beta}} \boldsymbol{\beta}]\left[\begin{array}{c}
\frac{\partial}{\partial \overline{\bar{\beta}}} \\
-\frac{\partial}{\partial \boldsymbol{\alpha}}
\end{array}\right] \exp \left[-\frac{1}{2}\left[\begin{array}{ll}
\overline{\boldsymbol{\beta}} & \boldsymbol{\alpha}
\end{array}\right](\underline{\underline{\mu}}+\underline{\underline{J}})\left[\begin{array}{c}
\boldsymbol{\alpha} \\
\overline{\boldsymbol{\beta}}
\end{array}\right]\right]\left[\begin{array}{ll}
\overline{\boldsymbol{\beta}} & \boldsymbol{\alpha}
\end{array}\right] \\
& =\exp [-\overline{\boldsymbol{\alpha}} \boldsymbol{\alpha}-\overline{\boldsymbol{\beta}} \boldsymbol{\beta}]\left[\underline{\underline{J}}(\underline{\underline{\mu}}+\underline{\underline{J}})\left[\begin{array}{c}
\boldsymbol{\alpha} \\
\overline{\boldsymbol{\beta}}
\end{array}\right]\left[\begin{array}{ll}
\overline{\boldsymbol{\beta}} & \boldsymbol{\alpha}
\end{array}\right]-\underline{\underline{J}}\right] \\
& \times \exp \left[-\frac{1}{2}\left[\begin{array}{ll}
\overline{\boldsymbol{\beta}} & \boldsymbol{\alpha}
\end{array}\right](\underline{\underline{\mu}}+\underline{\underline{J}})\left[\begin{array}{c}
\boldsymbol{\alpha} \\
\overline{\boldsymbol{\beta}}
\end{array}\right]\right] \text {. }
\end{aligned}
$$

Hence, using integration by parts and the above identities, (B.1) is obtained.

Next, we wish to express the above identities in terms of Majorana operators. Thus without changing the order of the ladder operators, we use the identities that relate the Majorana operators with the ladder operators given in (9), as well as the expression given in (11). We also multiply the right side of both sides of equation (B.1) by $2{\underline{\underline{U_{0}}}}^{-1}$, obtaining:

$$
\begin{aligned}
\left\{{\underline{\underline{U_{0}}}}^{-1} \hat{\underline{\gamma}}: \underline{\underline{\hat{\gamma}}}^{T} \hat{\Lambda}^{(u)}:\right\}= & \int \mathrm{d} \underline{\mathrm{\gamma}} \underline{\mathrm{\beta}} \mathrm{d} \underline{\alpha} \mathrm{d} \underline{\epsilon}|\boldsymbol{\gamma}\rangle\langle\boldsymbol{\epsilon}| \exp \left[-\overline{\boldsymbol{\alpha}} \boldsymbol{\alpha}-\overline{\boldsymbol{\beta}} \boldsymbol{\beta}-\frac{1}{2} \overline{\boldsymbol{\gamma}} \boldsymbol{\gamma}-\frac{1}{2} \bar{\epsilon} \boldsymbol{\epsilon}+\overline{\boldsymbol{\gamma}} \boldsymbol{\beta}+\overline{\boldsymbol{\alpha}} \boldsymbol{\epsilon}\right] \\
& \times\left[-\mathrm{i} 2 \underline{\underline{J}}{\underline{\underline{U_{0}}}}^{-1}(2 \underline{\underline{Y}}+\underline{\underline{\mathcal{I}}}) \underline{\underline{U_{0}}}\left[\begin{array}{c}
\boldsymbol{\alpha} \\
\overline{\boldsymbol{\beta}}
\end{array}\right]\left[\begin{array}{ll}
\overline{\boldsymbol{\beta}} & \boldsymbol{\alpha}
\end{array}\right]{\underline{\underline{U_{0}}}}^{-1}-2 \underline{\underline{J}} \underline{\underline{U_{0}}}{ }^{-1}\right] \\
& \times \exp \left[\frac{\mathrm{i}}{2}\left[\begin{array}{ll}
\overline{\boldsymbol{\beta}} & \boldsymbol{\alpha}
\end{array}\right]{\underline{\underline{U_{0}}}}^{-1}(2 \underline{\underline{Y}}+\underline{\underline{\mathcal{I}}}) \underline{\underline{U_{0}}}\left[\begin{array}{c}
\boldsymbol{\alpha} \\
\overline{\boldsymbol{\beta}}
\end{array}\right]\right]
\end{aligned}
$$


We notice that the second and third lines of (B.4) can be written in terms of the derivative of the un-normalized Gaussian operator itself with respect to $\underline{\underline{Y}}$. Hence we get the following result:

$$
\begin{aligned}
& \left\{{\underline{\underline{U_{0}}}}^{-1} \underline{\hat{\gamma}}: \underline{\hat{\underline{\gamma}}}^{T} \hat{\Lambda}^{(u)}:\right\}=\int \mathrm{d} \underline{\gamma} \mathrm{d} \underline{\beta} \mathrm{d} \underline{\alpha} \mathrm{d} \underline{\epsilon}|\boldsymbol{\gamma}\rangle\langle\boldsymbol{\epsilon}| \exp \left[-\overline{\boldsymbol{\alpha}} \boldsymbol{\alpha}-\overline{\boldsymbol{\beta}} \boldsymbol{\beta}-\frac{1}{2} \overline{\boldsymbol{\gamma}} \boldsymbol{\gamma}-\frac{1}{2} \overline{\boldsymbol{\epsilon}} \boldsymbol{\epsilon}+\overline{\boldsymbol{\gamma}} \boldsymbol{\beta}+\overline{\boldsymbol{\alpha}} \boldsymbol{\epsilon}\right] \\
& \times\left[\overline{\underline{I}}{\underline{\underline{U_{0}}}}^{-1}(2 \underline{\underline{Y}}+\underline{\underline{I}}) \frac{\mathrm{d}}{\mathrm{d} \underline{\underline{Y}}}-2 \underline{\underline{I}}{\underline{\underline{U_{0}}}}^{-1}\right] \\
& \times \exp \left[\frac{\mathrm{i}}{2}\left[\begin{array}{ll}
\overline{\boldsymbol{\beta}} & \boldsymbol{\alpha}
\end{array}\right]{\underline{\underline{U_{0}}}}^{-1}(2 \underline{\underline{Y}}+\underline{\underline{\mathcal{I}}}) \underline{\underline{\underline{U_{0}}}}\left[\begin{array}{l}
\boldsymbol{\alpha} \\
\overline{\boldsymbol{\beta}}
\end{array}\right]\right] \\
& =N\left[\underline{\underline{\underline{I}}}{\underline{\underline{U_{0}}}}^{-1}(2 \underline{\underline{Y}}+\underline{\underline{I}}) \frac{\mathrm{d}}{\mathrm{d} \underline{\underline{Y}}}-2 \underline{\underline{\underline{I}}}{\underline{\underline{U_{0}}}}^{-1}\right] \hat{\Lambda}^{(u)}(\underline{\underline{Y}}) \text {. }
\end{aligned}
$$

In order to obtain the last line of (B.6) we have used the resolution of unity of coherent states given in (B.2) and the inner product of fermionic coherent states given in (B.3) in order to express the Majorana Gaussian operator as:

$$
\begin{aligned}
\hat{\Lambda}^{(u)}(\underline{\underline{Y}}) \quad & =\int \mathrm{d} \underline{\gamma} \mathrm{d} \underline{\beta} \mathrm{d} \underline{\alpha} \mathrm{d} \underline{\epsilon}|\gamma\rangle\langle\boldsymbol{\epsilon}| \exp \left[-\overline{\boldsymbol{\alpha}} \boldsymbol{\alpha}-\overline{\boldsymbol{\beta}} \boldsymbol{\beta}-\frac{1}{2} \bar{\gamma} \boldsymbol{\gamma}-\frac{1}{2} \overline{\boldsymbol{\epsilon}} \boldsymbol{\epsilon}+\overline{\boldsymbol{\gamma}} \boldsymbol{\beta}+\overline{\boldsymbol{\alpha}} \boldsymbol{\epsilon}\right] \\
& \times \exp \left[\frac{\mathrm{i}}{2}\left[\begin{array}{ll}
\overline{\boldsymbol{\beta}} & \boldsymbol{\alpha}
\end{array}\right]{\underline{\underline{U_{0}}}}^{-1}(2 \underline{\underline{Y}}+\underline{\underline{\mathcal{I}}}) \underline{\underline{\underline{U_{0}}}}\left[\begin{array}{c}
\boldsymbol{\alpha} \\
\overline{\boldsymbol{\beta}}
\end{array}\right]\right] .
\end{aligned}
$$

On multiplying on the left both sides of (B.6), by $\underline{\underline{U_{0}}}$, we get:

$$
\begin{aligned}
\left\{\underline{\underline{\underline{\gamma}}}: \hat{\underline{\underline{\underline{T}}}}^{T} \hat{\Lambda}^{(u)}:\right\} & =\left[\underline{\underline{\underline{U_{0}}}} \underline{\underline{\underline{\underline{U}}}} \underline{\underline{\underline{U_{0}}}}{ }^{-1}(2 \underline{\underline{Y}}+\underline{\underline{\underline{I}}}) \frac{\mathrm{d}}{\mathrm{d} \underline{\underline{Y}}}-2 \underline{\underline{\underline{U_{0}}}} \underline{\underline{\underline{\underline{U}}}}{\underline{\underline{U_{0}}}}^{-1}\right] \hat{\Lambda}^{(u)} \\
& =\mathrm{i}\left[-\underline{\underline{\underline{I}}}(2 \underline{\underline{Y}}+\underline{\underline{I}}) \frac{\mathrm{d}}{\mathrm{d} \underline{\underline{Y}}}+2 \underline{\underline{I}}\right] \hat{\Lambda}^{(u)} .
\end{aligned}
$$

Here we have used that from the definition of $\underline{\underline{\mathcal{I}}}$ given in (14) we get $-i \underline{\underline{\mathcal{I}}}=\underline{\underline{U_{0}}} \underline{\underline{\underline{I}}}{\underline{\underline{U_{0}}}}^{-1}$. Therefore we have proved the differential identity given in (33).

Appendix B.2. Normally ordered products

$$
: \underline{\hat{\gamma}}^{T} \hat{\Lambda}^{(u)}:=\mathrm{i} \frac{d}{d \underline{\underline{Y}}} \hat{\Lambda}^{(u)}
$$

Proof. Analogous to the previous case we make use of the eigenvalue property of the fermionic coherent states as well as the completeness identity and the inner product of coherent states. Thus, we get:

$$
\begin{aligned}
: \underline{\widehat{a} \underline{a}}^{\dagger} \hat{\Lambda}^{(u)}:= & \int \mathrm{d} \underline{\gamma} \mathrm{d} \underline{\beta} \mathrm{d} \underline{\alpha} \mathrm{d} \underline{\epsilon}|\gamma\rangle\langle\boldsymbol{\epsilon}| \exp \left[-\overline{\boldsymbol{\alpha}} \boldsymbol{\alpha}-\overline{\boldsymbol{\beta}} \boldsymbol{\beta}-\frac{1}{2} \overline{\boldsymbol{\gamma}} \boldsymbol{\gamma}-\frac{1}{2} \overline{\boldsymbol{\epsilon}} \boldsymbol{\epsilon}+\overline{\boldsymbol{\gamma}} \boldsymbol{\beta}+\overline{\boldsymbol{\alpha}} \boldsymbol{\epsilon}\right] \\
& \times\left[\begin{array}{c}
\boldsymbol{\alpha} \\
\overline{\boldsymbol{\beta}}
\end{array}\right]\left[\begin{array}{ll}
\overline{\boldsymbol{\beta}} & \boldsymbol{\alpha}
\end{array}\right] \exp \left[-\frac{1}{2}\left[\begin{array}{ll}
\overline{\boldsymbol{\beta}} & \boldsymbol{\alpha}
\end{array}\right](\underline{\underline{\mu}}+\underline{\underline{J}})\left[\begin{array}{c}
\boldsymbol{\alpha} \\
\overline{\boldsymbol{\beta}}
\end{array}\right]\right] .
\end{aligned}
$$


Next, without changing the order of the Grassmann variables, we can write the above expression in terms of Majorana operators using equations (9) and (11). We also multiply both sides of the above equation on the left by $2 \underline{\underline{U_{0}}}$ and on the right by ${\underline{\underline{U_{0}}}}^{-1}$, thus obtaining:

$$
\begin{aligned}
& : \underline{\hat{\gamma}}^{T} \hat{\Lambda}^{(u)}: \quad=\int \mathrm{d} \underline{\gamma} \underline{\mathrm{d}} \underline{\beta} \mathrm{d} \underline{\alpha} \mathrm{d} \underline{\epsilon}|\boldsymbol{\gamma}\rangle\langle\boldsymbol{\epsilon}| \exp \left[-\overline{\boldsymbol{\alpha}} \boldsymbol{\alpha}-\overline{\boldsymbol{\beta}} \boldsymbol{\beta}-\frac{1}{2} \overline{\boldsymbol{\gamma}} \boldsymbol{\gamma}-\frac{1}{2} \overline{\boldsymbol{\epsilon}} \boldsymbol{\epsilon}+\bar{\gamma} \boldsymbol{\beta}+\overline{\boldsymbol{\alpha}} \boldsymbol{\epsilon}\right] \\
& \times 2 \underline{\underline{U_{0}}}\left[\begin{array}{l}
\boldsymbol{\alpha} \\
\overline{\boldsymbol{\beta}}
\end{array}\right]\left[\begin{array}{ll}
\overline{\boldsymbol{\beta}} & \boldsymbol{\alpha}
\end{array}\right]{\underline{\underline{U_{0}}}}^{-1} \exp \left[\frac{\mathrm{i}}{2}\left[\begin{array}{ll}
\overline{\boldsymbol{\beta}} & \boldsymbol{\alpha}
\end{array}\right]{\underline{\underline{U_{0}}}}^{-1}(2 \underline{\underline{Y}}+\underline{\underline{I}}) \underline{\underline{U_{0}}}\left[\begin{array}{l}
\boldsymbol{\alpha} \\
\overline{\boldsymbol{\beta}}
\end{array}\right]\right] \\
& =\int \mathrm{d} \underline{\gamma} \mathrm{d} \underline{\beta} \mathrm{d} \underline{\alpha} \mathrm{d} \underline{\epsilon}|\gamma\rangle\langle\boldsymbol{\epsilon}| \exp \left[-\overline{\boldsymbol{\alpha}} \boldsymbol{\alpha}-\overline{\boldsymbol{\beta}} \boldsymbol{\beta}-\frac{1}{2} \overline{\boldsymbol{\gamma}} \boldsymbol{\gamma}-\frac{1}{2} \overline{\boldsymbol{\epsilon}} \boldsymbol{\epsilon}+\overline{\boldsymbol{\gamma}} \boldsymbol{\beta}+\overline{\boldsymbol{\alpha}} \boldsymbol{\epsilon}\right] \\
& \times\left(\frac{d}{d \underline{\underline{Y}}} \exp \left[\frac{\mathrm{i}}{2}\left[\begin{array}{ll}
\overline{\boldsymbol{\beta}} & \boldsymbol{\alpha}
\end{array}\right]{\underline{\underline{U_{0}}}}^{-1}(2 \underline{\underline{Y}}+\underline{\underline{\underline{I}}}) \underline{\underline{\underline{U_{0}}}}\left[\begin{array}{l}
\boldsymbol{\alpha} \\
\overline{\boldsymbol{\beta}}
\end{array}\right]\right]\right) . \\
& =\mathrm{i} \frac{d}{d \underline{\underline{Y}}} \hat{\Lambda}^{(u)} \text {. }
\end{aligned}
$$

Here we have expressed the results of the first two lines as the derivative of the Gaussian operator an we have used the expression of the Majorana Gaussian operator given in (B.7). Therefore we have proved the differential identity given in (34).

Appendix B.3. Anti-normally ordered products

$$
\left\{\underline{\underline{\widehat{\gamma}}} \hat{\Lambda}^{T} \hat{\Lambda}^{(u)}\right\}=\underline{\mathrm{i} \mathcal{I}}\left\{(2 \underline{\underline{Y}}+\underline{\underline{I}}) \frac{\mathrm{d}}{\mathrm{d}} \underline{\underline{\underline{Y}}} \hat{\Lambda}^{u}-2 \hat{\Lambda}^{u}\right\}(2 \underline{\underline{Y}}+\underline{\underline{I}}) \underline{\underline{I}} .
$$

Proof. As in the previous cases we use the eigenvalue property of the fermionic coherent states as well as the completeness identity and the inner product of coherent states in order to write $\left\{\underline{a}^{\dagger} \hat{\Lambda}^{(u)}\right\}$ in terms of Grassmann variables, obtaining:

$$
\begin{aligned}
\left\{\underline{\widehat{a} \widehat{a}^{\dagger}} \hat{\Lambda}^{(u)}\right\}= & \int \underline{\mathrm{d}} \underline{\gamma} \mathrm{d} \underline{\beta} \mathrm{d} \underline{\alpha} \mathrm{d} \underline{\epsilon}|\gamma\rangle\left\langle\gamma\left|\left\{\underline{\widehat{a}}^{\dagger}|\boldsymbol{\beta}\rangle\left\langle\boldsymbol{\beta}\left|\widehat{\Lambda}^{(u)}\right| \boldsymbol{\alpha}\right\rangle\langle\boldsymbol{\alpha}|:\right\}\right| \boldsymbol{\epsilon}\right\rangle\langle\boldsymbol{\epsilon}| \\
= & \int \mathrm{d} \underline{\gamma} \mathrm{d} \underline{\beta} \mathrm{d} \underline{\alpha} \mathrm{d} \underline{\epsilon}|\gamma\rangle\langle\boldsymbol{\epsilon}| \exp \left[-\overline{\boldsymbol{\alpha}} \boldsymbol{\alpha}-\overline{\boldsymbol{\beta}} \boldsymbol{\beta}-\frac{1}{2} \overline{\boldsymbol{\gamma}} \boldsymbol{\gamma}-\frac{1}{2} \overline{\boldsymbol{\epsilon}} \boldsymbol{\epsilon}+\overline{\boldsymbol{\gamma}} \boldsymbol{\beta}+\overline{\boldsymbol{\alpha}} \boldsymbol{\epsilon}\right] \\
& \times \exp \left[-\frac{1}{2}\left[\begin{array}{ll}
\overline{\boldsymbol{\beta}} & \boldsymbol{\alpha}
\end{array}\right](\underline{\underline{\mu}}+\underline{\underline{J}})\left[\begin{array}{c}
\boldsymbol{\alpha} \\
\overline{\boldsymbol{\beta}}
\end{array}\right]\right]\left[\begin{array}{c}
\boldsymbol{\beta} \\
\overline{\boldsymbol{\alpha}}
\end{array}\right]\left[\begin{array}{ll}
\overline{\boldsymbol{\alpha}} & \boldsymbol{\beta}
\end{array}\right] .
\end{aligned}
$$

Next we wish to express the variables $\left[\begin{array}{c}\boldsymbol{\beta} \\ \overline{\boldsymbol{\alpha}}\end{array}\right]$ and $\left[\begin{array}{ll}\overline{\boldsymbol{\alpha}} & \boldsymbol{\beta}\end{array}\right]$ in the form given in the exponential of the Gaussian operator. In this case we will also use integration by parts but it will be performed twice. To this end we will use the following identities, which make use of Grassmann calculus:

$$
\exp \left[-\frac{1}{2}\left[\begin{array}{ll}
\overline{\boldsymbol{\beta}} & \boldsymbol{\alpha}
\end{array}\right](\underline{\underline{\mu}}+\underline{\underline{J}})\left[\begin{array}{c}
\boldsymbol{\alpha} \\
\overline{\boldsymbol{\beta}}
\end{array}\right]\right]\left[\begin{array}{c}
\boldsymbol{\beta} \\
\overline{\boldsymbol{\alpha}}
\end{array}\right]\left[\begin{array}{ll}
\overline{\boldsymbol{\alpha}} & \boldsymbol{\beta}
\end{array}\right] \exp [-\overline{\boldsymbol{\alpha}} \boldsymbol{\alpha}-\overline{\boldsymbol{\beta}} \boldsymbol{\beta}]=
$$


Phase space methods for Majorana fermions

$$
\begin{aligned}
& \exp \left[-\frac{1}{2}\left[\begin{array}{ll}
\overline{\boldsymbol{\beta}} & \boldsymbol{\alpha}
\end{array}\right](\underline{\underline{\mu}}+\underline{\underline{J}})\left[\begin{array}{c}
\boldsymbol{\alpha} \\
\overline{\boldsymbol{\beta}}
\end{array}\right]\right]\left[\begin{array}{c}
-\frac{\partial}{\partial \overline{\boldsymbol{\beta}}} \\
\frac{\partial}{\partial \boldsymbol{\alpha}}
\end{array}\right]\left[\begin{array}{ll}
\frac{\partial}{\partial \boldsymbol{\alpha}} & -\frac{\partial}{\partial \overline{\boldsymbol{\beta}}}
\end{array}\right] \exp [-\overline{\boldsymbol{\alpha}} \boldsymbol{\alpha}-\overline{\boldsymbol{\beta}} \boldsymbol{\beta}] \\
& \exp [-\overline{\boldsymbol{\alpha}} \boldsymbol{\alpha}-\overline{\boldsymbol{\beta}} \boldsymbol{\beta}]\left[\begin{array}{c}
-\frac{\partial}{\partial \overline{\boldsymbol{\beta}}} \\
\frac{\partial}{\partial \boldsymbol{\alpha}}
\end{array}\right]\left[\begin{array}{ll}
\frac{\partial}{\partial \boldsymbol{\alpha}} & -\frac{\partial}{\partial \overline{\boldsymbol{\beta}}}
\end{array}\right] \exp \left[-\frac{1}{2}\left[\begin{array}{ll}
\overline{\boldsymbol{\beta}} & \boldsymbol{\alpha}
\end{array}\right](\underline{\underline{\mu}}+\underline{\underline{J}})\left[\begin{array}{c}
\boldsymbol{\alpha} \\
\overline{\boldsymbol{\beta}}
\end{array}\right]\right] \\
& =\exp [-\overline{\boldsymbol{\alpha}} \boldsymbol{\alpha}-\overline{\boldsymbol{\beta}} \boldsymbol{\beta}]\left[\underline{\underline{J}}(\underline{\underline{\mu}}+\underline{\underline{J}})\left[\begin{array}{c}
\boldsymbol{\alpha} \\
\overline{\boldsymbol{\beta}}
\end{array}\right]\left[\begin{array}{ll}
\overline{\boldsymbol{\beta}} & \boldsymbol{\alpha}
\end{array}\right]-\underline{\underline{J}}\right](\underline{\underline{\mu}}+\underline{\underline{J}}) \underline{\underline{J}} \\
& \times \exp \left[-\frac{1}{2}\left[\begin{array}{ll}
\overline{\boldsymbol{\beta}} & \boldsymbol{\alpha}
\end{array}\right](\underline{\underline{\mu}}+\underline{\underline{J}})\left[\begin{array}{c}
\boldsymbol{\alpha} \\
\overline{\boldsymbol{\beta}}
\end{array}\right]\right] \text {. }
\end{aligned}
$$

The above expressions allow us to perform an integration by parts twice in (B.9) obtaining:

$$
\begin{aligned}
\left\{{\underline{\widehat{a} \widehat{a}^{\dagger}}}^{\hat{\Lambda}}{ }^{(u)}\right\}= & \int \mathrm{d} \underline{\underline{\gamma}} \underline{\mathrm{d}} \underline{\mathrm{d}} \underline{\mathrm{\alpha}} \underline{\mathrm{d}} \underline{\epsilon}|\boldsymbol{\gamma}\rangle\langle\boldsymbol{\epsilon}| \exp \left[-\overline{\boldsymbol{\alpha}} \boldsymbol{\alpha}-\overline{\boldsymbol{\beta}} \boldsymbol{\beta}-\frac{1}{2} \bar{\gamma} \boldsymbol{\gamma}-\frac{1}{2} \overline{\boldsymbol{\epsilon}} \boldsymbol{\epsilon}+\overline{\boldsymbol{\gamma}} \boldsymbol{\beta}+\overline{\boldsymbol{\alpha}} \boldsymbol{\epsilon}\right] \\
& \times\left[\underline{\underline{J}}(\underline{\underline{\mu}}+\underline{\underline{J}})\left[\begin{array}{c}
\boldsymbol{\alpha} \\
\overline{\boldsymbol{\beta}}
\end{array}\right]\left[\begin{array}{ll}
\overline{\boldsymbol{\beta}} & \boldsymbol{\alpha}
\end{array}\right]-\underline{\underline{J}}\right](\underline{\underline{\mu}}+\underline{\underline{J}}) \underline{\underline{J}} \\
& \times \exp \left[-\frac{1}{2}\left[\begin{array}{ll}
\overline{\boldsymbol{\beta}} & \boldsymbol{\alpha}
\end{array}\right](\underline{\underline{\mu}}+\underline{\underline{J}})\left[\begin{array}{c}
\boldsymbol{\alpha} \\
\overline{\boldsymbol{\beta}}
\end{array}\right]\right] .
\end{aligned}
$$

Next since we wish to express the identity in terms of Majorana operators and an anti-symmetric matrix, without changing the order of the ladder operators, we use the identities that relate the ladder operators with the Majorana operators given in (9), as well the relation given in (11) and (14). We also multiply both sides of the above equation on the left by $2 \underline{\underline{U_{0}}}$ and on the right by $\underline{\underline{U_{0}}}{ }^{-1}$, thus obtaining:

$$
\begin{aligned}
& \left\{\underline{\widehat{\gamma}}^{T} \hat{\Lambda}^{(u)}\right\}=\int \mathrm{d} \underline{\gamma} \underline{\mathrm{d}} \underline{\beta} \mathrm{d} \underline{\alpha} \mathrm{d} \underline{\epsilon}|\boldsymbol{\gamma}\rangle\langle\boldsymbol{\epsilon}| \exp \left[-\overline{\boldsymbol{\alpha}} \boldsymbol{\alpha}-\overline{\boldsymbol{\beta}} \boldsymbol{\beta}-\frac{1}{2} \bar{\gamma} \boldsymbol{\gamma}-\frac{1}{2} \overline{\boldsymbol{\epsilon}} \boldsymbol{\epsilon}+\overline{\boldsymbol{\gamma}} \boldsymbol{\beta}+\overline{\boldsymbol{\alpha}} \boldsymbol{\epsilon}\right] \\
& \times 2\left[\underline{\underline{I}}(2 \underline{\underline{Y}}+\underline{\underline{I}}) \underline{\underline{\underline{U_{0}}}}\left[\begin{array}{c}
\boldsymbol{\alpha} \\
\overline{\boldsymbol{\beta}}
\end{array}\right]\left[\begin{array}{ll}
\overline{\boldsymbol{\beta}} & \boldsymbol{\alpha}
\end{array}\right]{\underline{\underline{U_{0}}}}^{-1}-\underline{\mathrm{i \mathcal {I }}}\right](2 \underline{\underline{Y}}+\underline{\underline{I}}) \underline{\underline{I}} \\
& \times \exp \left[\frac{\mathrm{i}}{2}\left[\begin{array}{ll}
\overline{\boldsymbol{\beta}} & \boldsymbol{\alpha}
\end{array}\right]{\underline{\underline{U_{0}}}}^{-1}(2 \underline{\underline{Y}}+\underline{\underline{\underline{I}}}) \underline{\underline{U_{0}}}\left[\begin{array}{c}
\boldsymbol{\alpha} \\
\overline{\boldsymbol{\beta}}
\end{array}\right]\right] .
\end{aligned}
$$

As in the previous cases we notice that the second and third line of the above expressions can be expressed in terms of the derivative of the un-normalized Gaussian operator with respect to $\underline{\underline{Y}}$. We also use the expression for the un-normalized Gaussian operators given in (B.7). Therefore we get:

$$
\left\{{\underline{\widehat{\gamma} \hat{\gamma}^{T}}}^{\hat{\Lambda}^{(u)}}\right\}=\mathrm{i} \underline{\underline{I}}\left\{(2 \underline{\underline{Y}}+\underline{\underline{I}}) \frac{\mathrm{d}}{\mathrm{d} \underline{\underline{Y}}} \hat{\Lambda}^{u}-2 \hat{\Lambda}^{u}\right\}(2 \underline{\underline{Y}}+\underline{\underline{I}}) \underline{\underline{I}} .
$$

Thus we have proved the differential identity given in (35)). 
Phase space methods for Majorana fermions

\section{Appendix C. Normalized Majorana differential identities}

Here we consider the normalized Gaussian operators given in (19). We also used the change of variables given in (36).

Appendix C.1. First mixed product

$$
\left\{\underline{\hat{\hat{\gamma}}}: \underline{\hat{\gamma}}^{T} \hat{\Lambda}:\right\}=\mathrm{i}(\underline{\underline{X}}+\underline{\underline{I}}) \frac{\mathrm{d} \hat{\Lambda}(\underline{\underline{X}})}{\mathrm{d}} \underline{\underline{\underline{X}}}(\underline{\underline{X}}-\underline{\underline{I}})-\mathrm{i}(\underline{\underline{X}}-\underline{\underline{I}}) \hat{\Lambda}(\underline{\underline{X}}) .
$$

Proof. We make a change of variables using (36) on the differential identities given in (33) obtaining:

$\left\{\underline{\hat{\gamma}}: \underline{\hat{\gamma}}^{T} \hat{\Lambda}:\right\}=\mathrm{i} N(\underline{\underline{X}})\left[-\underline{\underline{\mathcal{I}}}\left(2(\underline{\underline{X}}-\underline{\underline{\mathcal{I}}})^{-1}-\underline{\underline{\underline{I}}}\right) \frac{\mathrm{d}}{\mathrm{d} \underline{\underline{Y}}}+2 \underline{\underline{\mathcal{I}}}\right] \hat{\Lambda}^{(u)}(\underline{\underline{X}})$.

Next, we use the chain rule in order to change the derivative, which is:

$\frac{\mathrm{d} \hat{\Lambda}^{(u)}}{\mathrm{d} \underline{\underline{Y}}}=\frac{\mathrm{d} \hat{\Lambda}^{(u)}}{\mathrm{d} \underline{\underline{X}}} \frac{\mathrm{d} \underline{\underline{\underline{X}}}}{\mathrm{~d}}=-(\underline{\underline{\underline{X}}}-\underline{\underline{I}}) \frac{\mathrm{d} \hat{\Lambda}^{(u)}}{\mathrm{d} \underline{\underline{X}}}(\underline{\underline{X}}-\underline{\underline{\mathcal{I}}})$.

On substituting the above identities in (C.1) and on simplifying terms we get:

$\left\{\underline{\hat{\gamma}}: \underline{\hat{\gamma}}^{T} \hat{\Lambda}:\right\}=\mathrm{i} N(\underline{\underline{X}})\left[2 \underline{\underline{\underline{I}}} \hat{\Lambda}^{(u)}(\underline{\underline{X}})+(\underline{\underline{X}}+\underline{\underline{I}}) \frac{d \hat{\Lambda}^{(u)}(\underline{\underline{X}})}{d \underline{\underline{X}}}(\underline{\underline{X}}-\underline{\underline{I}})\right]$.

We now wish to relate the derivative of the un-normalized Gaussian operator with the derivative of the normalized one. Hence we use the following relation:

$\frac{\mathrm{d} \hat{\Lambda}(\underline{\underline{X}})}{\mathrm{d} \underline{\underline{X}}}=\frac{\mathrm{d}}{\mathrm{d} \underline{\underline{X}}} N(\underline{\underline{X}}) \hat{\Lambda}^{(u)}(\underline{\underline{X}})=N(\underline{\underline{X}}) \hat{\Lambda}^{(u)}(\underline{\underline{X}})(\underline{\underline{X}}-\underline{\underline{I}})^{-1}+N(\underline{\underline{X}}) \frac{\mathrm{d} \hat{\Lambda}^{(u)}(\underline{\underline{X}})}{\mathrm{d} \underline{\underline{X}}}$.

Here we have used the following result for the derivative of the square root of the determinant of an antisymmetric matrix:

$\frac{\mathrm{d} N(\underline{\underline{X}})}{\mathrm{d} \underline{\underline{X}}}=\frac{\mathrm{d}}{\mathrm{d} \underline{\underline{X}}} \frac{1}{2^{M}} \sqrt{\operatorname{det}[\underline{\underline{X}}]}=\frac{1}{2^{M}} \sqrt{\operatorname{det}[\underline{\underline{X}}]}(\underline{\underline{X}}-\underline{\underline{\mathcal{I}}})^{-1}=N(\underline{\underline{X}})(\underline{\underline{X}}-\underline{\underline{I}})^{-1}$.

From (C.4) we get:

$$
\frac{\mathrm{d} \hat{\Lambda}^{(u)}(\underline{\underline{X}})}{\mathrm{d} \underline{\underline{X}}}=\frac{1}{N(\underline{\underline{X}})} \frac{\mathrm{d} \hat{\Lambda}(\underline{\underline{X}})}{\mathrm{d} \underline{\underline{X}}}-\hat{\Lambda}^{(u)}(\underline{\underline{X}})(\underline{\underline{X}}-\underline{\underline{\mathcal{I}}})^{-1} .
$$

On substituting this result on (C.3) and simplifying we get:

$$
\left\{\underline{\hat{\hat{\gamma}}}: \underline{\hat{\gamma}}^{T} \hat{\Lambda}:\right\}=\mathrm{i}(\underline{\underline{X}}+\underline{\underline{\underline{I}}}) \frac{\mathrm{d} \hat{\Lambda}(\underline{\underline{X}})}{\mathrm{d} \underline{\underline{X}}}(\underline{\underline{X}}-\underline{\underline{I}})-\mathrm{i}(\underline{\underline{X}}-\underline{\underline{I}}) \hat{\Lambda}(\underline{\underline{X}}) \text {. }
$$

Therefore we have proved the differential identity given in (37). 
Appendix C.2. Second mixed product

$$
: \hat{\hat{\gamma}}\left\{\underline{\hat{\gamma}}^{T} \hat{\Lambda}\right\}:=\mathrm{i}\left[\underline{\underline{X}}^{-} \frac{\mathrm{d} \hat{\Lambda}}{\mathrm{d} \underline{\underline{X}}}-\hat{\Lambda}\right] \underline{\underline{X}}^{+}
$$

Proof. We can derive this fourth mixed identity from the third one. We know the orderings of both identities as given in (A.1 and A.2). Utilizing those orderings and Fermi commutation relation in (1) we get:

$$
: \underline{\hat{\gamma}}\left\{\underline{\hat{\gamma}}^{T} \hat{\Lambda}\right\}:=-\left\{\underline{\hat{\gamma}}: \underline{\hat{\gamma}}^{T} \hat{\Lambda}:\right\}^{T}-2 i \underline{\hat{\Lambda} \mathcal{I}}
$$

This will give us the new fourth identity as in (38)

Appendix C.3. Normally ordered products

$$
: \underline{\hat{\gamma} \hat{\gamma}^{T}} \hat{\Lambda}:=\mathrm{i}(\underline{\underline{X}}-\underline{\underline{I}}) \hat{\Lambda}(\underline{\underline{X}})-\mathrm{i}(\underline{\underline{X}}-\underline{\underline{I}}) \frac{\mathrm{d} \hat{\Lambda}(\underline{\underline{X}})}{\mathrm{d} \underline{\underline{X}}}(\underline{\underline{X}}-\underline{\underline{I}}) \text {. }
$$

Proof. We use $\hat{\Lambda}(\underline{\underline{X}})=N(\underline{\underline{X}}) \hat{\Lambda}^{(u)}(\underline{\underline{X}})$ and we also use the expressions given in (C.2) and (C.5) in order to express the derivative in terms of the normalized Gaussian operators which is

$$
\frac{\mathrm{d}}{\mathrm{d} \underline{\underline{Y}}} \hat{\Lambda}^{(u)}=-\frac{1}{N(\underline{\underline{X}})}(\underline{\underline{X}}-\underline{\underline{I}}) \frac{\mathrm{d} \hat{\Lambda}(\underline{\underline{X}})}{\mathrm{d} \underline{\underline{X}}}(\underline{\underline{X}}-\underline{\underline{I}})+(\underline{\underline{X}}-\underline{\underline{I}}) \hat{\Lambda}^{(u)}(\underline{\underline{X}}) \text {. }
$$

On substituting the above result in (34) we get:

$$
: \underline{\hat{\gamma} \hat{\gamma}^{T}} \hat{\Lambda}:=\mathrm{i}(\underline{\underline{X}}-\underline{\underline{I}}) \hat{\Lambda}(\underline{\underline{X}})-\mathrm{i}(\underline{\underline{X}}-\underline{\underline{I}}) \frac{\mathrm{d} \hat{\Lambda}(\underline{\underline{X}})}{\mathrm{d} \underline{\underline{X}}}(\underline{\underline{X}}-\underline{\underline{I}}) \text {. }
$$

Thus we have proved the differential identity given in (39).

Appendix C.4. Anti-normally ordered products

$$
\left\{\underline{\widehat{\gamma}}^{T} \hat{\Lambda}\right\}=\mathrm{i}\left[-(\underline{\underline{\mathcal{I}}}+\underline{\underline{X}}) \frac{\mathrm{d} \hat{\Lambda}(\underline{\underline{X}})}{\mathrm{d} \underline{\underline{X}}}(\underline{\underline{\mathcal{I}}}+\underline{\underline{X}})+\hat{\Lambda}(\underline{\underline{X}})(\underline{\underline{\mathcal{I}}}+\underline{\underline{X}})\right]
$$

Proof. As in the previous cases we use $\hat{\Lambda}(\underline{\underline{X}})=N(\underline{\underline{X}}) \hat{\Lambda}^{(u)}(\underline{\underline{X}})$ and we also make a change of variables using (36) on the differential identities given in (35) obtaining:

$$
\left\{\underline{\widehat{\gamma}}^{T} \hat{\Lambda}\right\}=\mathrm{i} N(\underline{\underline{X}}) \underline{\underline{I}}\left\{\left(2(\underline{\underline{X}}-\underline{\underline{I}})^{-1}-\underline{\underline{I}}\right) \frac{\mathrm{d}}{\mathrm{d} \underline{\underline{Y}}}-2\right\} \hat{\Lambda}^{(u)}(\underline{\underline{X}})\left(2(\underline{\underline{X}}-\underline{\underline{I}})^{-1}-\underline{\underline{I}}\right) \underline{\underline{I}} .
$$

Next, we express the derivative in terms of the Gaussian operators and the $\underline{\underline{X}}$ matrix using the identity given in (C.6) obtaining:

$$
\begin{aligned}
\left\{\underline{\widehat{\gamma}}^{T} \hat{\Lambda}\right\}= & \underline{\mathrm{i}} \underline{\underline{I}}\left\{\left(2(\underline{\underline{X}}-\underline{\underline{I}})^{-1}-\underline{\underline{I}}\right)\left[-(\underline{\underline{X}}-\underline{\underline{I}}) \frac{\mathrm{d} \hat{\Lambda}(\underline{\underline{X}})}{\mathrm{d} \underline{\underline{X}}}(\underline{\underline{X}}-\underline{\underline{I}})+(\underline{\underline{X}}-\underline{\underline{I}}) \hat{\Lambda}(\underline{\underline{X}})\right]\right. \\
& -2 \hat{\Lambda}(\underline{\underline{X}})\}\left(2(\underline{\underline{X}}-\underline{\underline{I}})^{-1}-\underline{\underline{I}}\right) \underline{\underline{I}} .
\end{aligned}
$$


On simplifying terms and using that $\underline{\underline{\mathcal{I I}}}=-\underline{\underline{I}}$ we get:

$$
\left\{\underline{\widehat{\gamma}}^{T} \hat{\Lambda}\right\}=\mathrm{i}\left[-(\underline{\underline{\mathcal{I}}}+\underline{\underline{X}}) \frac{\mathrm{d} \hat{\Lambda}(\underline{\underline{X}})}{\mathrm{d} \underline{\underline{X}}}(\underline{\underline{\mathcal{I}}}+\underline{\underline{X}})+\hat{\Lambda}(\underline{\underline{X}})(\underline{\underline{\mathcal{I}}}+\underline{\underline{X}})\right]
$$

Therefore we have proved the differential identity given in (40).

\section{Appendix D. Majorana differential identities for unordered products}

In this Section we give the detailed calculations for unordered Majorana differential identities. These are derived using the ordered identities given in Appendix C.

Appendix D.1. Unordered left and right products

$$
\underline{\hat{\gamma} \hat{\gamma}^{T}} \hat{\Lambda}=\mathrm{i}\left[(1+\underline{\mathrm{i}} \underline{\underline{x}}) \frac{d \hat{\Lambda}}{d \underline{\underline{x}}}(1-\underline{\mathrm{i}} \underline{\underline{x}})-\hat{\Lambda}(\underline{\underline{x}}+\mathrm{i})\right] \text {. }
$$

Proof. The explicit expression for $\hat{\gamma} \hat{\gamma}^{T} \hat{\Lambda}$ in terms of the Fermi operators is given in (A.5). The idea is to find the appropriate combination between the four different expressions of the ordered products of Majorana operators and the Gaussian operator that gives the expression given in (A.5). Each ordered product will be used in order to obtain the products of only Fermi creation operators with the Gaussian operators, or Fermi annihilation operators and the Gaussian operators or the two different product of Fermi creation and annihilation operator with the Gaussian operator in the left

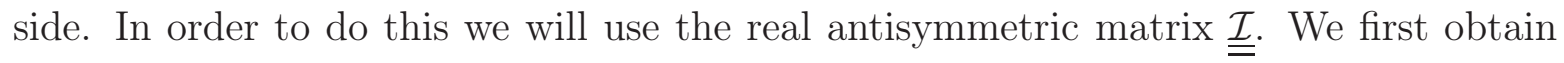
the combination of the anti-normal product that will give the matrix form with only product of Fermi annihilation operators and the Gaussian one. Hence on pre and post multiplying (A.4) by $\underline{\underline{I}}$, we get:

$$
\begin{aligned}
& \underline{\mathcal{I}}\left\{\hat{\gamma}_{\mu} \hat{\gamma}_{v} \hat{\Lambda}\right\} \underline{\mathcal{I}}= \\
& \left(\begin{array}{cc}
\widehat{a}_{i} \widehat{a}_{j} \hat{\Lambda}+\hat{\Lambda} \widehat{a}_{i}^{\dagger} \widehat{a}_{j}^{\dagger}-\widehat{a}_{i} \hat{\Lambda} \widehat{a}_{j}^{\dagger}+\widehat{a}_{j} \hat{\Lambda} \widehat{a}_{i}^{\dagger} & -i \widehat{a}_{i} \widehat{a}_{j} \hat{\Lambda}+i \hat{\Lambda} \widehat{a}_{i}^{\dagger} \widehat{a}_{j}^{\dagger}-i \widehat{a}_{i} \hat{\Lambda} \widehat{a}_{j}^{\dagger}-i \widehat{a}_{j} \hat{\Lambda} \widehat{a}_{i}^{\dagger} \\
-i \widehat{a}_{i} \widehat{a}_{j} \hat{\Lambda}+i \hat{\Lambda} \widehat{a}_{i}^{\dagger} \widehat{a}_{j}^{\dagger}+i \widehat{a}_{i} \hat{\Lambda} \widehat{a}_{j}^{\dagger}+i \widehat{a}_{j} \hat{\Lambda} \widehat{a}_{i}^{\dagger} & -\widehat{a}_{i} \widehat{a}_{j} \hat{\Lambda}-\hat{\Lambda} \widehat{a}_{i}^{\dagger} \widehat{a}_{j}^{\dagger}-\widehat{a}_{i} \hat{\Lambda} \widehat{a}_{j}^{\dagger}+\widehat{a}_{j} \hat{\Lambda} \widehat{a}_{i}^{\dagger}
\end{array}\right) .
\end{aligned}
$$

Now adding (A.4) and (D.2) leads to:

$$
B_{1}=\left(\begin{array}{cc}
2 \widehat{a}_{i} \widehat{a}_{j} \hat{\Lambda}+2 \hat{\Lambda} \widehat{a}_{i}^{\dagger} \widehat{a}_{j}^{\dagger} & -2 \mathrm{i} \widehat{a}_{i} \widehat{a}_{j} \hat{\Lambda}+2 \mathrm{i} \hat{\Lambda} \widehat{a}_{i}^{\dagger} \widehat{a}_{j}^{\dagger} \\
-2 \mathrm{i} \widehat{a}_{i} \widehat{a}_{j} \hat{\Lambda}+2 \mathrm{i} \hat{\Lambda} \widehat{a}_{i}^{\dagger} \widehat{a}_{j}^{\dagger} & -2 \widehat{a}_{i} \widehat{a}_{j} \hat{\Lambda}-2 \hat{\Lambda} \widehat{a}_{i}^{\dagger} \widehat{a}_{j}^{\dagger}
\end{array}\right),
$$

where $B_{1}=\left\{\hat{\gamma}_{\mu} \hat{\gamma}_{v} \hat{\Lambda}\right\}+\underline{\underline{I}}\left\{\hat{\gamma}_{\mu} \hat{\gamma}_{v} \hat{\Lambda}\right\} \underline{\underline{I}}$. We notice that we can obtain the required product of Fermi annihilation operators and $\hat{\Lambda}$ from the following expression:

$$
\frac{B_{1}-\mathrm{i} B_{1} \underline{\underline{I}}}{4}=\left(\begin{array}{cc}
\widehat{a}_{i} \widehat{a}_{j} \hat{\Lambda} & -i \widehat{a}_{i} \widehat{a}_{j} \hat{\Lambda} \\
-i \widehat{a}_{i} \widehat{a}_{j} \hat{\Lambda} & -\widehat{a}_{i} \widehat{a}_{j} \hat{\Lambda}
\end{array}\right)
$$


In a similar way we wish to obtain a matrix of the product of Fermi creation operators with $\hat{\Lambda}$. In this case we consider (A.3) and perform the same procedures as described above obtaining:

$$
B_{2}=\left(\begin{array}{cc}
2 \widehat{a}_{i}^{\dagger} \widehat{a}_{j}^{\dagger} \hat{\Lambda}+2 \hat{\Lambda} \widehat{a}_{i} \widehat{a}_{j} & -\mathrm{i}\left[-2 \widehat{a}_{i}^{\dagger} \widehat{a}_{j}^{\dagger} \hat{\Lambda}+2 \hat{\Lambda} \widehat{a}_{i} \widehat{a}_{j}\right] \\
-\mathrm{i}\left[-2 \widehat{a}_{i}^{\dagger} \widehat{a}_{j}^{\dagger} \hat{\Lambda}+2 \hat{\Lambda} \widehat{a}_{i} \widehat{a}_{j}\right] & -2 \widehat{a}_{i}^{\dagger} \widehat{a}_{j}^{\dagger} \hat{\Lambda}-2 \hat{\Lambda} \widehat{a}_{i} \widehat{a}_{j}
\end{array}\right),
$$

where $B_{2}=: \hat{\gamma}_{\mu} \hat{\gamma}_{v} \hat{\Lambda}:+\underline{\underline{\underline{\mathcal{I}}}}: \hat{\gamma}_{\mu} \hat{\gamma}_{v} \hat{\Lambda}: \underline{\underline{\underline{\mathcal{I}}}}$. Then the required expression is:

$$
\frac{B_{2}+\mathrm{i} B_{2} \underline{\underline{I}}}{4}=\left(\begin{array}{cc}
\widehat{a}_{i}^{\dagger} \widehat{a}_{j}^{\dagger} \hat{\Lambda} & \mathrm{i} \widehat{a}_{i}^{\dagger} \widehat{a}_{j}^{\dagger} \hat{\Lambda} \\
\mathrm{i} \widehat{a}_{i}^{\dagger} \widehat{a}_{j}^{\dagger} \hat{\Lambda} & -\widehat{a}_{i}^{\dagger} \widehat{a}_{j}^{\dagger} \hat{\Lambda}
\end{array}\right)
$$

Similarly, we use the mixed product given in (A.1) in order to obtain:

$$
C_{1}=\left(\begin{array}{cc}
2 \widehat{a}_{i} \widehat{a}_{j}^{\dagger} \hat{\Lambda}-2 \hat{\Lambda} \widehat{a}_{j} \widehat{a}_{i}^{\dagger} & \mathrm{i}\left[2 \widehat{a}_{i} \widehat{a}_{j}^{\dagger} \hat{\Lambda}+2 \hat{\Lambda} \widehat{a}_{j} \widehat{a}_{i}^{\dagger}\right] \\
\mathrm{i}\left[-2 \widehat{a}_{i} \widehat{a}_{j}^{\dagger} \hat{\Lambda}-2 \hat{\Lambda} \widehat{a}_{j} \widehat{a}_{i}^{\dagger}\right] & 2 \widehat{a}_{i} \widehat{a}_{j}^{\dagger} \widehat{\Lambda}-2 \widehat{\Lambda} \widehat{a}_{j} \widehat{a}_{i}^{\dagger}
\end{array}\right),
$$

where $C_{1}=\left\{\hat{\gamma}_{\mu}: \hat{\gamma}_{v} \hat{\Lambda}:\right\}-\underline{\underline{I}}\left\{\hat{\gamma}_{\mu}: \hat{\gamma}_{v} \hat{\Lambda}:\right\} \underline{\underline{I}}$ and from this expression we get a matrix in terms of product of creation and annihilation operators and $\hat{\Lambda}$ :

$$
\frac{C_{1}+\mathrm{i} C_{1} \underline{\underline{\underline{I}}}}{4}=\left(\begin{array}{cc}
\widehat{a}_{i} \widehat{a}_{j}^{\dagger} \hat{\Lambda} & \mathrm{i} \widehat{a}_{i} \widehat{a}_{j}^{\dagger} \hat{\Lambda} \\
-\mathrm{i} \widehat{a}_{i} \widehat{a}_{j}^{\dagger} \hat{\Lambda} & \widehat{a}_{i} \widehat{a}_{j}^{\dagger} \widehat{\Lambda}
\end{array}\right) .
$$

Following the same steps as described above from (A.2) we get:

$$
C_{2}=\left(\begin{array}{cc}
2 \widehat{a}_{i}^{\dagger} \widehat{a}_{j} \hat{\Lambda}-2 \hat{\Lambda} \widehat{a}_{j}^{\dagger} \widehat{a}_{i} & \mathrm{i}\left[-2 \widehat{a}_{i}^{\dagger} \widehat{a}_{j} \hat{\Lambda}-2 \hat{\Lambda} \widehat{a}_{j}^{\dagger} \widehat{a}_{i}\right] \\
\mathrm{i}\left[+2 \widehat{a}_{i}^{\dagger} \widehat{a}_{j} \hat{\Lambda}+2 \hat{\Lambda} \widehat{a}_{j}^{\dagger} \widehat{a}_{i}\right] & 2 \widehat{a}_{i}^{\dagger} \widehat{a}_{j} \hat{\Lambda}-2 \hat{\Lambda} \widehat{a}_{j}^{\dagger} \widehat{a}_{i}
\end{array}\right),
$$

where: $C_{2}=: \hat{\gamma}_{\mu}\left\{\hat{\gamma}_{v} \hat{\Lambda}\right\}:-\underline{\underline{I}}: \hat{\gamma}_{\mu}\left\{\hat{\gamma}_{v} \hat{\Lambda}\right\}: \underline{\underline{I}}$ and we also obtain:

$$
\frac{C_{2}-\mathrm{i} C_{2} \underline{\underline{I}}}{4}=\left(\begin{array}{cc}
\widehat{a}_{i}^{\dagger} \widehat{a}_{j} \hat{\Lambda} & -i \widehat{a}_{i}^{\dagger} \widehat{a}_{j} \hat{\Lambda} \\
i \widehat{a}_{i}^{\dagger} \widehat{a}_{j} \hat{\Lambda} & \widehat{a}_{i}^{\dagger} \widehat{a}_{j} \hat{\Lambda}
\end{array}\right) .
$$

On adding the identities given in (D.5), (D.4), (D.6), and (D.7) we obtain the combination of Fermi operators and the Gaussian operators corresponding to the unordered product of Majorana operators and $\hat{\Lambda}$ given in (A.5):

$$
\underline{\hat{\gamma}}^{T} \hat{\Lambda}=\frac{C_{1}+\mathrm{i} C_{1} \underline{\underline{\underline{I}}}}{4}+\frac{C_{2}-\mathrm{i} C_{2} \underline{\underline{\underline{I}}}}{4}+\frac{B_{1}-\mathrm{i} B_{1} \underline{\underline{\underline{I}}}}{4}+\frac{B_{2}+\mathrm{i} B_{2} \underline{\underline{\mathcal{I}}}}{4} .
$$

In order to obtain the differential identity we notice that $B_{1}, B_{2}, C_{1}$ and $C_{2}$ and given in terms of the normalized ordered differential identities derived in Appendix C. Thus using the expression in the right hand side of the four normalized differential identities and on simplifying terms we get:

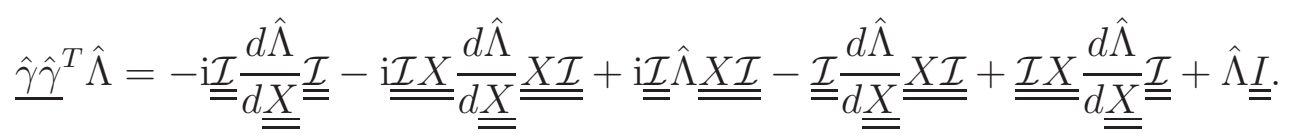


The above equation can be written in a simple form as:

$$
\underline{\hat{\gamma} \hat{\gamma}^{T}} \hat{\Lambda}=-\underline{\mathrm{i} \mathcal{I}}\left[(1+i \underline{\underline{X}}) \frac{d \hat{\Lambda}}{d \underline{\underline{X}}}(1-\underline{\underline{\mathrm{X}}})-\mathrm{i} \hat{\Lambda}(1-\underline{\underline{\mathrm{X}}})\right] \underline{\underline{\mathcal{I}}} .
$$

In terms of the alternate antisymmetric form, $\underline{\underline{x}}=\underline{\underline{\mathcal{I}} X^{T}} \underline{\underline{I}}$, we can define:

$$
\frac{d \hat{\Lambda}}{d \underline{\underline{x}}^{T}}=\underline{\underline{\mathcal{I}}} \frac{d \hat{\Lambda}}{d \underline{\underline{X}}} \underline{\underline{I}}
$$

In this way we can introduce $\underline{\underline{x}}^{ \pm}=\underline{\underline{x}} \pm \underline{\mathrm{i}} \underline{\underline{I}}$, and simplify (D.8) to give:

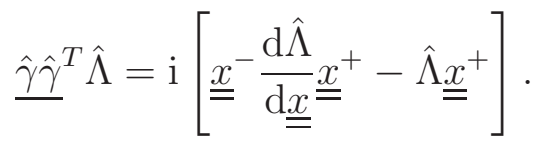

On conjugating this expression, we obtain the right product identity:

$$
\hat{\Lambda} \underline{\hat{\gamma} \hat{\gamma}^{T}}=\mathrm{i}\left[\underline{\underline{x}}^{+} \frac{\mathrm{d} \hat{\Lambda}}{\mathrm{d} \underline{\underline{x}}} \underline{\underline{x}}^{-}-\hat{\Lambda} \underline{\underline{x}}^{-}\right] \text {. }
$$

Appendix D.2. Unordered mixed products

$$
\underline{\hat{\gamma}} \hat{\Lambda} \underline{\hat{\gamma}}^{T}=\mathrm{i}\left[(\underline{\underline{i x}}+\underline{\underline{I}}) \frac{d \hat{\Lambda}}{d \underline{\underline{x}}}(\underline{\underline{I}}+\underline{\mathrm{i}} \underline{\underline{x}})-i \hat{\Lambda}(\underline{\underline{I}}+\mathrm{i} \underline{\underline{x}})\right] .
$$

Proof. Analogous to the previous differential identity, the method is to use the four different orderings of products of Majorana operators and Gaussian operators to give an explicit expression for $\hat{\hat{\gamma}} \hat{\Lambda} \hat{\gamma}^{T}$, in terms of the ordered Fermi operators and Gaussian operators. Next we transform this into the corresponding differential identities.

First, we obtain matrices of products of the Fermi creation and annihilation operators where the Gaussian operator is in the middle. Thus, pre and post multiplying (A.4) by the real antisymmetric matrix $\underline{\underline{I}}$, we get:

$$
\begin{aligned}
& \underline{\underline{I}}\left\{\hat{\gamma}_{\mu} \hat{\gamma}_{v} \hat{\Lambda}\right\} \underline{I}= \\
& \left(\begin{array}{cc}
\widehat{a}_{i} \widehat{a}_{j} \hat{\Lambda}+\hat{\Lambda} \widehat{a}_{i}^{\dagger} \widehat{a}_{j}^{\dagger}-\widehat{a}_{i} \hat{\Lambda} \widehat{a}_{j}^{\dagger}+\widehat{a}_{j} \hat{\Lambda} \widehat{a}_{i}^{\dagger} & -i \widehat{a}_{i} \widehat{a}_{j} \hat{\Lambda}+i \hat{\Lambda} \widehat{a}_{i}^{\dagger} \widehat{a}_{j}^{\dagger}-i \widehat{a}_{i} \hat{\Lambda} \widehat{a}_{j}^{\dagger}-i \widehat{a} \widehat{a}_{j} \hat{\Lambda} \widehat{a}_{i}^{\dagger} \\
-i \widehat{a}_{i} \widehat{a}_{j} \hat{\Lambda}+i \hat{\Lambda} \widehat{a}_{i}^{\dagger} \widehat{a}_{j}^{\dagger}+i \widehat{a}_{i} \hat{\Lambda} \widehat{a}_{j}^{\dagger}+i \widehat{a}_{j} \hat{\Lambda} \widehat{a}_{i}^{\dagger} & -\widehat{a}_{i} \widehat{a}_{j} \hat{\Lambda}-\hat{\Lambda} \widehat{a}_{i}^{\dagger} \widehat{a}_{j}^{\dagger}-\widehat{a}_{i} \hat{\Lambda} \widehat{a}_{j}^{\dagger}+\widehat{a}_{j} \hat{\Lambda} \widehat{a}_{i}^{\dagger}
\end{array}\right),(D
\end{aligned}
$$

Adding (A.4) and (D.12) leads to:

$$
D_{1}=\left(\begin{array}{cc}
2 \widehat{a}_{i} \hat{\Lambda} \widehat{a}_{j}^{\dagger}-2 \widehat{a}_{j} \hat{\Lambda} \widehat{a}_{i}^{\dagger} & 2 i \widehat{a}_{i} \hat{\Lambda} \widehat{a}_{j}^{\dagger}+2 i \widehat{a}_{j} \hat{\Lambda} \widehat{a}_{i}^{\dagger} \\
-2 i \widehat{a}_{i} \hat{\Lambda} \widehat{a}_{j}^{\dagger}-2 i \widehat{a}_{j} \hat{\Lambda} \widehat{a}_{i}^{\dagger} & 2 \widehat{a}_{i} \hat{\Lambda} \widehat{a}_{j}^{\dagger}-2 \widehat{a}_{j} \hat{\Lambda} \widehat{a}_{i}^{\dagger}
\end{array}\right),
$$

where $D_{1}=\left\{\hat{\gamma}_{\mu} \hat{\gamma}_{v} \hat{\Lambda}\right\}-\underline{\underline{I}}\left\{\hat{\gamma}_{\mu} \hat{\gamma}_{v} \hat{\Lambda}\right\} \underline{\mathcal{I}}$. Using this expression we obtain:

$$
\frac{D_{1}+\mathrm{i} D_{1} \underline{\underline{I}}}{4}=\left(\begin{array}{cc}
\widehat{a}_{i} \hat{\Lambda} \widehat{a}_{j}^{\dagger} & \mathrm{i} \widehat{a}_{i} \hat{\Lambda} \widehat{a}_{j}^{\dagger} \\
-\mathrm{i} \widehat{a}_{i} \hat{\Lambda} \widehat{a}_{j}^{\dagger} & \widehat{a}_{i} \hat{\Lambda} \widehat{a}_{j}^{\dagger}
\end{array}\right)
$$


In this case, using the anti-normal ordering identity we obtain a matrix with products of the form $\widehat{a}_{i} \hat{\Lambda} \widehat{a}_{j}^{\dagger}$. We now use the the same procedure as described above for the normal ordering product, (A.3), obtaining:

$$
D_{2}=\left(\begin{array}{cc}
-2 \widehat{a}_{j}^{\dagger} \hat{\Lambda} \widehat{a}_{i}+2 \widehat{a}_{i}^{\dagger} \hat{\Lambda} \widehat{a}_{j} & -i 2 \widehat{a}_{j}^{\dagger} \hat{\Lambda} \widehat{a}_{i}-i 2 \widehat{a}_{i}^{\dagger} \hat{\Lambda} \widehat{a}_{j} \\
i 2 \widehat{a}_{j}^{\dagger} \hat{\Lambda} \widehat{a}_{i}+i 2 \widehat{a}_{i}^{\dagger} \hat{\Lambda} \widehat{a}_{j} & -2 \widehat{a}_{j}^{\dagger} \hat{\Lambda} \widehat{a}_{i}+2 \widehat{a}_{i}^{\dagger} \hat{\Lambda} \widehat{a}_{j}
\end{array}\right),
$$

where $D_{2}=: \hat{\gamma}_{\mu} \hat{\gamma}_{v} \hat{\Lambda}:-\underline{\underline{I}}: \hat{\gamma}_{\mu} \hat{\gamma}_{v} \hat{\Lambda}: \underline{\underline{I}}$. From this we get a matrix with products of the form $\widehat{a}_{i}^{\dagger} \hat{\Lambda} \widehat{a}_{j}$ :

$$
\frac{D_{2}-\mathrm{i} D_{2} \underline{\underline{\underline{I}}}}{4}=\left(\begin{array}{cc}
\widehat{a}_{i}^{\dagger} \hat{\Lambda} \widehat{a}_{j} & -\mathrm{i} \widehat{a}_{i}^{\dagger} \hat{\Lambda} \widehat{a}_{j} \\
\mathrm{i} \widehat{a}_{i}^{\dagger} \hat{\Lambda} \widehat{a}_{j} & \widehat{a}_{i}^{\dagger} \hat{\Lambda} \widehat{a}_{j}
\end{array}\right) .
$$

Next, using (A.1) we arrive at the following expression:

$$
\frac{E_{1}-\mathrm{i} E_{1} \underline{\underline{\underline{\mathcal{I}}}}}{4}=\left(\begin{array}{cc}
\widehat{a}_{i} \hat{\Lambda} \widehat{a}_{j} & -\mathrm{i} \widehat{a}_{i} \hat{\Lambda} \widehat{a}_{j} \\
-\mathrm{i} \widehat{a}_{i} \hat{\Lambda} \widehat{a}_{j} & -\widehat{a}_{i} \hat{\Lambda} \widehat{a}_{j}
\end{array}\right)
$$

where $E_{1}=\left\{\hat{\gamma}_{\mu}: \hat{\gamma}_{v} \hat{\Lambda}:\right\}+\underline{\underline{I}}\left\{\hat{\gamma}_{\mu}: \hat{\gamma}_{v} \hat{\Lambda}:\right\} \underline{\underline{I}}$.

Using (A.2) we obtain a matrix with products of the form $\widehat{a}_{i}^{\dagger} \hat{\Lambda} \widehat{a}_{j}^{\dagger}$ :

$$
\frac{E_{2}+\mathrm{i} E_{2} \underline{\underline{\underline{\mathcal{I}}}}}{4}=\left(\begin{array}{cc}
\widehat{a}_{i}^{\dagger} \hat{\Lambda} \widehat{a}_{j}^{\dagger} & \mathrm{i} \widehat{a}_{i}^{\dagger} \hat{\Lambda} \widehat{a}_{j}^{\dagger} \\
\mathrm{i} \widehat{a}_{i}^{\dagger} \hat{\Lambda} \widehat{a}_{j}^{\dagger} & -\widehat{a}_{i}^{\dagger} \hat{\Lambda} \widehat{a}_{j}^{\dagger}
\end{array}\right),
$$

where: $E_{2}=: \hat{\gamma}_{\mu}\left\{\hat{\gamma}_{v} \hat{\Lambda}\right\}:+\underline{\underline{\mathcal{I}}}: \hat{\gamma}_{\mu}\left\{\hat{\gamma}_{v} \hat{\Lambda}\right\}: \underline{\underline{\mathcal{I}}}$.

On adding the expressions given in (D.15), (D.14), (D.16), and (D.17) we get the appropriate combination of products of Fermi operators and the Gaussian operator:

$$
\underline{\hat{\gamma}} \hat{\Lambda} \underline{\hat{\gamma}}^{T}=\frac{E_{1}-\mathrm{i} E_{1} \underline{\underline{\underline{\mathcal{I}}}}}{4}+\frac{E_{2}+\mathrm{i} E_{2} \underline{\underline{\underline{\mathcal{I}}}}}{4}+\frac{D_{1}+\mathrm{i} D_{1} \underline{\underline{\underline{\mathcal{I}}}}}{4}+\frac{D_{2}-\mathrm{i} D_{2} \underline{\underline{\underline{\mathcal{I}}}}}{4} .
$$

Now utilizing the right hand side of the four normalized differential identities an on simplifying terms we arrive at the following expression:

$$
\underline{\hat{\gamma}} \underline{\hat{\Lambda}} \underline{\hat{\gamma}}^{T}=-i \underline{\underline{\underline{I}}} \frac{d \hat{\Lambda}}{d \underline{\underline{X}}} \underline{\underline{\mathcal{I}}}+i \underline{\underline{\underline{I} X}} \frac{d \hat{\Lambda}}{d \underline{\underline{X}}} \underline{\underline{X \mathcal{I}}}-i \underline{\underline{\mathcal{I}}} \underline{\underline{\underline{X \mathcal{I}}}}+\underline{\underline{\underline{I}}} \frac{d \hat{\Lambda}}{d \underline{\underline{X}}} \underline{\underline{X \mathcal{I}}}+\underline{\underline{\mathcal{I} X}} \frac{d \hat{\Lambda}}{d \underline{\underline{X}}} \underline{\underline{\mathcal{I}}}+\hat{\underline{\Lambda}} \underline{\underline{I}} .
$$

Taking common terms outside we get:

$$
\underline{\hat{\hat{\gamma}}} \underline{\underline{\hat{\gamma}}}{ }^{T}=-i \underline{\underline{\mathcal{I}}}\left[(i \underline{\underline{X}}+1) \frac{d \hat{\Lambda}}{d \underline{\underline{X}}}(1+i \underline{\underline{X}})-i \hat{\Lambda}(1+i \underline{\underline{X}})\right] \underline{\underline{\mathcal{I}}} .
$$

This leads to our final result, which can also be written as:

$$
\underline{\hat{\gamma}} \hat{\hat{\Lambda}} \underline{\hat{\gamma}}^{T}=\mathrm{i}\left[-\underline{\underline{x}}^{-} \frac{\mathrm{d} \hat{\Lambda}}{\mathrm{d} \underline{\underline{x}}} \underline{\underline{\underline{x}}}+\underline{\underline{\Lambda}} \underline{\underline{x}}^{-}\right] .
$$




\section{Appendix E. Time evolution of the Majorana Q-function}

Here we provide the details of the calculations in order to derive (71), (72) and (73) of Section 5. The chain rule allow us to obtain the relation between the Gaussian basis $\hat{\Lambda}^{N}(\underline{\underline{x}})$ and the Gaussian operator $\hat{\Lambda}(\underline{\underline{x}})$, which is:

$$
\frac{1}{\mathcal{N}} S\left([\underline{\underline{x}}]^{2}\right) \frac{\mathrm{d} \hat{\Lambda}(\underline{\underline{x}})}{\mathrm{d} \underline{\underline{x}}}=\frac{\mathrm{d} \hat{\Lambda}^{N}(\underline{\underline{x}})}{\mathrm{d} \underline{\underline{x}}}-\frac{\mathrm{d} \ln S\left([\underline{\underline{x}}]^{2}\right)}{\mathrm{d} \underline{\underline{x}}} \hat{\Lambda}^{N}(\underline{\underline{x}}) .
$$

Using the above identity in (70) and the definition of the Majorana Q-function we get:

$$
\begin{aligned}
\frac{\mathrm{d} Q(\underline{\underline{x}})}{\mathrm{d} t}= & -\Omega_{\mu \nu}\left[-x_{\mu \kappa} \frac{\mathrm{d} Q}{\mathrm{~d} x_{v \kappa}}+x_{\mu \kappa} \frac{\mathrm{d} \ln S\left([\underline{\underline{x}}]^{2}\right)}{\mathrm{d} x_{v \kappa}}\right] \\
& -\Omega_{\mu \nu}\left[\frac{\mathrm{d} Q}{\mathrm{~d} x_{\kappa \mu}} x_{\kappa v}-\frac{\mathrm{d} \ln S\left([\underline{\underline{x}}]^{2}\right)}{\mathrm{d} x_{\kappa \mu}} x_{\kappa v}\right] .
\end{aligned}
$$

Here we have used the following convention for calculation matrix derivatives $[\mathrm{d} / \mathrm{d} x]_{\kappa v}=$ $\mathrm{d} / \mathrm{d} x_{v \kappa}[36$.

In the limit $S\left([\underline{\underline{x}}]^{2}\right) \rightarrow 1$, the above expression reduces to (71). Next, using the chain rule we obtain the following expressions:

$$
\begin{aligned}
-x_{\mu \kappa} \frac{\mathrm{d} Q}{\mathrm{~d} x_{v \kappa}} & =-\frac{\mathrm{d}}{\mathrm{d} x_{v \kappa}}\left(x_{\mu \kappa} Q\right)+\left(\frac{\mathrm{d}}{\mathrm{d} x_{v \kappa}} x_{\mu \kappa}\right) Q \\
& =-\frac{\mathrm{d}}{\mathrm{d} x_{v \kappa}}\left(x_{\mu \kappa} Q\right)+(2 M-1) \delta_{\mu v} Q,
\end{aligned}
$$

and

$$
\begin{aligned}
\frac{\mathrm{d} Q}{\mathrm{~d} x_{\kappa \mu}} x_{\kappa v} & =\frac{\mathrm{d}}{\mathrm{d} x_{\kappa \mu}}\left(Q x_{\kappa v}\right)-\left(\frac{\mathrm{d}}{\mathrm{d} x_{\kappa \mu}} x_{\kappa v}\right) Q \\
& =\frac{\mathrm{d}}{\mathrm{d} x_{\kappa \mu}}\left(Q x_{\kappa v}\right)-(2 M-1) \delta_{v \mu} Q .
\end{aligned}
$$

On substituting (E.3 and E.4) in (71) we get (72). The method of characteristics allow us to solve the above equation as:

$$
\frac{\mathrm{d} x_{v \kappa}}{\mathrm{d} t}=\Omega_{\nu \mu} x_{\mu \kappa}-x_{v \mu} \Omega_{\mu \kappa},
$$

where we used that $\underline{\underline{\Omega}}$ is an antisymmetric matrix. In matrix form the above equation is:

$$
\frac{\mathrm{d} \underline{\underline{x}}}{\mathrm{~d} t}=[\underline{\underline{\Omega}}, \underline{\underline{x}}] .
$$

Here (E.6) corresponds to (73). 


\section{Appendix F. Time evolution for the open quantum system}

In this section we show the details of the calculations in order to obtain the time evolution equation of the small quantum dot coupled to a zero temperature reservoir, for the multimode case. This equation is given in (86). The time evolution equation for the Q-function written in terms of products of Majorana variables and Majorana Gaussian operators is given in (83). This equation is:

$$
\begin{aligned}
\frac{\mathrm{d} Q}{\mathrm{~d} t}= & -\frac{i}{2} \frac{1}{\mathcal{N}} S \operatorname{Tr}\left[\tilde{\Omega}_{\kappa \nu}\left\{\underline{\hat{\gamma}}: \underline{\hat{\gamma}}^{T} \hat{\Lambda}:\right\}_{\nu \kappa} \hat{\rho}\right] \\
& -\frac{1}{2 i} \frac{1}{\mathcal{N}} S \operatorname{Tr}\left[\Upsilon_{\kappa \nu}\left(: \underline{\hat{\gamma}} \underline{\hat{\gamma}}^{T} \hat{\Lambda}:{ }_{\nu \kappa}-\left\{\underline{\hat{\gamma}}: \hat{\underline{\gamma}}^{T} \hat{\Lambda}:\right\}_{\nu \kappa}\right) \hat{\rho}\right]-\gamma_{i j} \delta_{i j} Q .
\end{aligned}
$$

We now use the Majorana differential identities given in (37) and (39) respectively. On simplifying terms and using identities corresponding identities we obtain:

$$
\begin{aligned}
\frac{\mathrm{d} Q}{\mathrm{~d} t} & =\frac{1}{2} \frac{1}{\mathcal{N}} S \operatorname{Tr}\left[\tilde{\Omega}_{\kappa \nu}\left[\underline{\underline{X}}+\frac{d \hat{\Lambda}(\underline{\underline{X}})}{d \underline{\underline{X}}} \underline{\underline{X}}^{-}-\underline{\underline{X}}^{-\hat{\Lambda}}(\underline{\underline{X}})\right]_{\nu \kappa} \hat{\rho}\right] \\
& +\frac{1}{\mathcal{N}} S \operatorname{Tr}\left[\Upsilon_{\kappa \nu}\left[\underline{\underline{X}} \frac{d \hat{\Lambda}(\underline{\underline{X}})}{d \underline{\underline{X}}} \underline{\underline{X}}^{-}\right]_{\nu \kappa} \hat{\rho}\right]-\Upsilon_{\kappa \nu} X_{\nu \kappa} Q
\end{aligned}
$$

provided $\gamma_{i j} \delta_{i j}=\Upsilon_{\kappa \nu} \mathcal{I}_{\nu \kappa}$. Due to the same reason as stated in the previous time evolution example, $\tilde{\Omega}_{\kappa \nu} X_{\nu \kappa}^{-}$will be zero. Now utilizing the chain rule (E.1), the definition of the Q-function and considering the limit $S\left([\underline{\underline{X}}]^{2}\right) \rightarrow 1$, we get:

$$
\frac{\mathrm{d} Q}{\mathrm{~d} t}=\frac{1}{2} \tilde{\Omega}_{\kappa \nu}\left[\underline{\underline{X}}^{+} \frac{\mathrm{d} Q}{\mathrm{~d} \underline{\underline{X}}} \underline{\underline{X}}^{-}\right]_{\nu \kappa}+\Upsilon_{\kappa \nu}\left[\underline{\underline{X}} \frac{\mathrm{d} Q}{\underline{\underline{X}}} \underline{\underline{X}}\right]_{\nu \kappa}-\Upsilon_{\kappa \nu} X_{\nu \kappa} Q
$$

Next, using the following result of the product rule:

$X_{\nu \ell} \frac{\mathrm{d} Q}{\mathrm{~d} X_{p \ell}} X_{p \kappa}^{-}=\frac{\mathrm{d}}{\mathrm{d} X_{p \ell}} X_{\nu \ell} X_{p \kappa}^{-} Q-\left(\delta_{\nu p} \delta_{\ell \ell}-\delta_{\nu \ell} \delta_{\ell p}\right) X_{p \kappa}^{-} Q-\left(\delta_{p p} \delta_{\kappa \ell}-\delta_{p \ell} \delta_{\kappa p}\right) X_{\nu \ell} Q$,

on the time evolution equation we finally obtain:

$$
\begin{aligned}
\frac{\mathrm{d} Q}{\mathrm{~d} t}= & \frac{1}{2} \tilde{\Omega}_{\kappa \nu} \frac{\mathrm{d}}{\mathrm{d} X_{p \ell}}\left(X_{\nu \ell}^{+} Q X_{p \kappa}^{-}\right)+\Upsilon_{\kappa \nu} \frac{\mathrm{d}}{\mathrm{d} X_{p \ell}}\left(X_{\nu \ell} X_{p \kappa}^{-} Q\right)-\Upsilon_{\kappa \nu} X_{\nu \kappa} Q \\
& -\Upsilon_{\kappa \nu}\left(\delta_{\nu p} \delta_{\ell \ell}-\delta_{\nu \ell} \delta_{\ell p}\right) X_{p \kappa}^{-} Q-\Upsilon_{\kappa \nu}\left(\delta_{p p} \delta_{\kappa \ell}-\delta_{p \ell} \delta_{\kappa p}\right) X_{\nu \ell} Q .
\end{aligned}
$$

Upon summation by considering all the $2 M$ modes, $\sum \delta_{\ell \ell}=2 M$, and on simplifying we finally get:

$$
\begin{aligned}
\frac{\mathrm{d} Q}{\mathrm{~d} t}= & \frac{1}{2} \tilde{\Omega}_{\kappa \nu} \frac{\mathrm{d}}{\mathrm{d} X_{p \ell}}\left(X_{\nu \ell}^{+} Q X_{p \kappa}^{-}\right)+\Upsilon_{\kappa \nu} \frac{\mathrm{d}}{\mathrm{d} X_{p l}}\left(X_{\nu \ell} Q X_{p \kappa}^{-}\right) \\
& -(4 M-1) X_{\nu \kappa} \Upsilon_{\kappa \nu} Q+(2 M-1) \Upsilon_{\kappa \nu} \mathcal{I}_{\nu \kappa} .
\end{aligned}
$$

This corresponds to (86). 


\section{References}

[1] Majorana E 1937 Il Nuovo Cimento 14 171-184

[2] Alicea J 2012 Reports on Progress in Physics 75076501

[3] Elliott S R and Franz M 2015 Rev. Mod. Phys. 87(1) 137-163

[4] Bloch I, Dalibard J and Zwerger W 2008 Rev. Mod. Phys. 80 885-964

[5] Fu L and Kane C L 2008 Phys. Rev. Lett. 100096407

[6] Mourik V, Zuo K, Frolov S M, Plissard S R, Bakkers E P A M and Kouwenhoven L P 2012 Science 336 1003-1007

[7] Nadj-Perge S, Drozdov I K, Li J, Chen H, Jeon S, Seo J, MacDonald A H, Bernevig B A and Yazdani A 2014 Science 346 602-607

[8] Xu J P, Wang M X, Liu Z L, Ge J F, Yang X, Liu C, Xu Z A, Guan D, Gao C L, Qian D, Liu Y, Wang Q H, Zhang F C, Xue Q K and Jia J F 2015 Phys. Rev. Lett. 114(1) 017001

[9] Rokhinson L P, Liu X and Furdyna J K 2012 Nat. Phys. 8 795-799

[10] Beenakker C W J 2013 Annual Review of Condensed Matter Physics 4 113-136

[11] Beenakker C W J 2015 Rev. Mod. Phys. 87(3) 1037-1066

[12] Das Sarma S, Freedman M and Nayak C 2015 Npj Quantum Information 115001

[13] Kitaev A Y 2001 Physics-Uspekhi 44131

[14] Stern A and Lindner N H 2013 Science 339 1179-1184

[15] Wang L, Liu Y H, Iazzi M, Troyer M and Harcos G 2015 Phys. Rev. Lett. 115(25) 250601

[16] Huffman E and Chandrasekharan S 2016 Phys. Rev. E 94(4) 043311

[17] Li Z X, Jiang Y F and Yao H 2015 Phys. Rev. B 91(24) 241117

[18] Li Z X, Jiang Y F and Yao H 2016 Phys. Rev. Lett. 117(26) 267002

[19] Wei Z C, Wu C, Li Y, Zhang S and Xiang T 2016 Phys. Rev. Lett. 116(25) 250601

[20] Terhal B M and DiVincenzo D P 2002 Phys. Rev. A 65032325

[21] Bravyi S and König R 2012 Communications in Mathematical Physics 316 641-692

[22] Pedrocchi F L, Bonesteel N E and DiVincenzo D P 2015 Phys. Rev. B 92115441

[23] Valiant L 2001 Proceedings of the 33rd ACM Symposium on the Theory of Computation (ACM Press) pp 114-123

[24] Weidlich W, Risken H and Haken H 1967 Z. Phys. A 204 223-239

[25] Hillery M, O'Connell R F, Scully M O and Wigner E P 1984 Phys. Rep. 106 121-167

[26] Gardiner C W and Zoller P 2000 Quantum Noise 2nd ed (Berlin: Springer)

[27] Blakie P B, Bradley A S, Davis M J, Ballagh R J and Gardiner C W 2008 Adv. Phys. 57 363-455

[28] Polkovnikov A 2010 Ann. Phys. 325 1790-1852

[29] Carmichael H J 1999 Statistical methods in quantum optics 1. Master Equations and Fokker-Planck Equations. (Berlin, London: Springer)

[30] Gardiner C W 1985 Stochastic Methods 1st ed (Berlin: Springer)

[31] Drummond P D and Chaturvedi S 2016 Physica Scripta 91073007

[32] Arecchi F T, Courtens E, Gilmore R and Thomas H 1972 Phys. Rev. A 6 2211-2237

[33] Gilmore R, Bowden C M and Narducci L M 1975 Phys. Rev. A 12 1019-1031

[34] Reid M D, Opanchuk B, Rosales-Zárate L and Drummond P D 2014 Phys. Rev. A 90012111

[35] Zambrini R, Barnett S, Colet P and San Miguel M 2003 Eur. Phys. J. D 22 461-471

[36] Corney J F and Drummond P D 2006 J. Phys. A 39 269-297

[37] Corney J F and Drummond P D 2006 Phys. Rev. B 73125112

[38] Corney J F and Drummond P D 2004 Phys. Rev. Lett. 93260401

[39] Aimi T and Imada M 2007 J. Phys. Soc. Jpn. 76084709

[40] Corboz P, Ögren M, Kheruntsyan K and Corney J F 2011 Phase-Space Methods for Fermions Quantum Gases: Finite Temperature and Non-Equilibrium Dynamics. Vol 1. Cold Atom Series ed Proukakis N, Gardiner S and Davis M (Imperial College Press)

[41] Ögren M, Kheruntsyan K and Corney J F 2010 EuroPhys. Lett. 9236003

[42] Ögren M, Kheruntsyan K and Corney J F 2011 Comput. Phys. Commun. 182 1999-2003 
[43] Rosales-Zárate L E C and Drummond P D 2015 New J. Phys. 17032002

[44] Altland A and Zirnbauer M R 1997 Phys. Rev. B 55 1142-1161

[45] Liu X J and Drummond P D 2012 Phys. Rev. A 86(3) 035602

[46] Lutchyn R M, Sau J D and Das Sarma S 2010 Phys. Rev. Lett. 105(7) 077001

[47] Bagrets D and Altland A 2012 Phys. Rev. Lett. 109(22) 227005

[48] DeGottardi W, Thakurathi M, Vishveshwara S and Sen D 2013 Phys. Rev. B 88(16) 165111

[49] Gibertini M, Fazio R, Polini M and Taddei F 2013 Phys. Rev. B 88(14) 140508

[50] Morimoto T and Furusaki A 2013 Phys. Rev. B 88(12) 125129

[51] Budich J C and Ardonne E 2013 Phys. Rev. B 88(7) 075419

[52] Chiu C K, Teo J C Y, Schnyder A P and Ryu S 2016 Rev. Mod. Phys. 88(3) 035005

[53] Hegde S S and Vishveshwara S 2016 Phys. Rev. B 94(11) 115166

[54] Aimi T and Imada M 2007 J. Phys. Soc. Jpn. 76113708

[55] Cahill K E and Glauber R J 1999 Phys. Rev. A 59 1538-1555

[56] Rosales-Zárate L E C and Drummond P D 2013 J. Phys. A 46275203

[57] Opanchuk B, Rosales-Zárate L, Reid M and Drummond P 2014 Physics Letters A 378946 - 949

[58] Dalton B J, Jeffers J and Barnett S M 2016 Fortschritte der Physik 65, 1600038

[59] Bogolyubov N 1958 Soviet Physics-JETP 751

[60] Valatin J 1958 Il Nuovo Cimento (1955-1965) 7 843-857

[61] Balian R and Brezin E 1969 Il Nuovo Cimento B 64 37-55

[62] Kraus C V, Wolf M M, Cirac J I and Giedke G 2009 Phys. Rev. A 79(1) 012306

[63] Eisler V and Zimborás Z 2015 New Journal of Physics 17053048

[64] Meichanetzidis K, Eisert J, Cirio M, Lahtinen V and Pachos J K 2016 Phys. Rev. Lett. 116(13) 130501

[65] Cartan E 1926 Bulletin de la S. M. F. 54 214-264

[66] Cartan E 1927 Bulletin de la S. M. F. 55 114-134

[67] Cartan E 1935 Abhandlungen aus dem Mathematischen Seminar der Universitat Hamburg 11(1) $116-162$

[68] Hua L K 1963 Harmonic analysis of functions of several complex variables in the classical domains (American Mathematical Society, Providence, Rhode Island)

[69] Helgason S 2001 Differential geometry, Lie groups, and symmetric spaces (Providence, R.I.: American Mathematical Society)

[70] Caselle M and Magnea U 2004 Phys. Rep. 394 41-156

[71] Xu Y 2005 Theory of complex homogeneous bounded domains (Beijing: Science Press; London: Kluwer Academic)

[72] Heinzner P, Huckleberry A and Zirnbauer M R 2005 Commun. Math. Phys. 257 725-771

[73] Mehta M L 2004 Random matrices 3rd ed (Academic Press, Boston)

[74] Forrester P J 2010 Log-gases and random matrices (Princeton: Princeton University Press)

[75] Prosen T 2008 New Journal of Physics 10043026

[76] Meichanetzidis K, Cirio M, Pachos J K and Lahtinen V 2016 Phys. Rev. B 94115158

[77] Kitaev A 2009 AIP Conference Proceedings 1134 22-30

[78] Husimi K 1940 Proc. Phys. Math. Soc. Jpn 22 264-314

[79] Drummond P D and Hillery M 2014 The Quantum Theory of Nonlinear Optics (Cambridge University Press) 